TRANSACTIONS OF THE

AMERICAN MATHEMATICAL SOCIETY

Volume 354, Number 11, Pages 4421-4454

S 0002-9947(02)03073-8

Article electronically published on July 2, 2002

\title{
EXTENSIONS FOR FINITE CHEVALLEY GROUPS II
}

\author{
CHRISTOPHER P. BENDEL, DANIEL K. NAKANO, AND CORNELIUS PILLEN
}

\begin{abstract}
Let $G$ be a semisimple simply connected algebraic group defined and split over the field $\mathbb{F}_{p}$ with $p$ elements, let $G\left(\mathbb{F}_{q}\right)$ be the finite Chevalley group consisting of the $\mathbb{F}_{q}$-rational points of $G$ where $q=p^{r}$, and let $G_{r}$ be the $r$ th Frobenius kernel. The purpose of this paper is to relate extensions between modules in $\operatorname{Mod}\left(G\left(\mathbb{F}_{q}\right)\right)$ and $\operatorname{Mod}\left(G_{r}\right)$ with extensions between modules in $\operatorname{Mod}(G)$. Among the results obtained are the following: for $r>2$ and $p \geq 3(h-1)$, the $G\left(\mathbb{F}_{q}\right)$-extensions between two simple $G\left(\mathbb{F}_{q}\right)$-modules are isomorphic to the $G$-extensions between two simple $p^{r}$-restricted $G$-modules with suitably "twisted" highest weights. For $p \geq 3(h-1)$, we provide a complete characterization of $\mathrm{H}^{1}\left(G\left(\mathbb{F}_{q}\right), H^{0}(\lambda)\right)$ where $H^{0}(\lambda)=\operatorname{ind}_{B}^{G} \lambda$ and $\lambda$ is $p^{r}$-restricted. Furthermore, for $p \geq 3(h-1)$, necessary and sufficient bounds on the size of the highest weight of a $G$-module $V$ are given to insure that the restriction map $\mathrm{H}^{1}(G, V) \rightarrow \mathrm{H}^{1}\left(G\left(\mathbb{F}_{q}\right), V\right)$ is an isomorphism. Finally, it is shown that the extensions between two simple $p^{r}$-restricted $G$-modules coincide in all three categories provided the highest weights are "close" together.
\end{abstract}

\section{INTRODUCTION}

1.1. Let $G$ be a semisimple simply connected algebraic group scheme defined and split over the field $\mathbb{F}_{p}$ with $p$ elements, and let $k$ be the algebraic closure of $\mathbb{F}_{p}$. Let $G\left(\mathbb{F}_{q}\right)$ be the finite Chevalley group consisting of $\mathbb{F}_{q}$-rational points of $G$ where $q=p^{r}$ for a nonnegative integer $r$. Moreover, let $G_{r}$ be the $r$ th Frobenius kernel. The relationship between the characters for simple modules in $\operatorname{Mod}(G), \operatorname{Mod}\left(G_{r}\right)$ and $\operatorname{Mod}\left(G\left(\mathbb{F}_{q}\right)\right)$ is well-understood. Yet, the characters and degrees of the simple modules in these categories are not known in general. Lusztig formulated a wellknown conjecture which would allow one to compute the characters of these simple modules by using Kahzdan-Lusztig polynomials when $p$ is larger than the Coxeter number. Cline, Parshall and Scott [CPS2] have demonstrated that Lusztig's conjecture can be reformulated by using the cohomology theory of $G$. They provide necessary and sufficient conditions to insure the validity of the Lusztig conjecture via a criterion involving nonvanishing of extensions between certain simple modules. Furthermore, if the Lusztig conjecture is established, then a calculation of $\operatorname{Ext}_{G}^{1}(L(\lambda), L(\mu))$ where $L(\lambda)$ and $L(\mu)$ are simple $G$-modules can be obtained. The purpose of this paper is to relate extensions between simple modules in $\operatorname{Mod}\left(G\left(\mathbb{F}_{q}\right)\right)$

Received by the editors November 16, 2001.

2000 Mathematics Subject Classification. Primary 20C33, 20G10; Secondary 20G05, 20J06.

Research of the second author was supported in part by NSF grant DMS-0102225.

(C)2002 American Mathematical Society 
and $\operatorname{Mod}\left(G_{r}\right)$ with extensions between simple modules in $\operatorname{Mod}(G)$. Such relationships are very important to understand for both conceptual as well as computational purposes. For most of our results, it is necessary to assume that the prime $p$ is "large" relative to the Coxeter number of the group.

The results in this paper are outlined as follows. In Section 2, we summarize several basic formulas developed in BNP2] which express $\operatorname{Ext}_{G\left(\mathbb{F}_{q}\right)}^{1}(L(\lambda), L(\mu))$ in terms of extensions for modules over $G$ and $G_{1}$. These formulas are refined further in Theorem 2.4 by using work of Andersen And1. This allows us to identify in Corollary 2.4 necessary conditions on the $p$-adic expansions of weights $\lambda$ and $\mu$ for the existence of nontrivial extensions of $L(\lambda)$ by $L(\mu)$ over $G$ or $G\left(\mathbb{F}_{q}\right)$. Moreover, these formulas also allow us to provide generalizations of Ye's results for rank 2 groups Ye1, Ye2, (see Theorem 2.5). Finally, we similarly obtain formulas expressing $\operatorname{Ext}_{G_{r}}^{1}(L(\lambda), L(\mu))$ in terms of extensions over $G$ and $G_{1}$.

At the beginning of Section 3, it is shown that for $r \geq 2$, one can compute all extensions between simple $G\left(\mathbb{F}_{q}\right)$-modules if one knows all extensions between simple $G$-modules. In particular, we succeed in showing that for $r \geq 3, \operatorname{Ext}_{G\left(\mathbb{F}_{q}\right)}^{1}(L(\lambda)$, $L(\mu)) \cong \operatorname{Ext}_{G}^{1}(L(\tilde{\lambda}), L(\tilde{\mu}))$ for suitably constructed weights $\tilde{\lambda}$ and $\tilde{\mu}$. Later in the section, we prove that there is also a one-to-one correspondence between extensions for simple modules over $G\left(\mathbb{F}_{q}\right)$ and certain extensions of simple modules with $p^{r+1}$-restricted highest weights over $G$. This improves earlier work of the third author.

In Section 4, our attention focuses on comparing the cohomology of modules over finite and algebraic groups. For $G$-modules $V$ with $p^{r}$-restricted composition factors, we show in Theorem 4.1 that one can determine $\mathrm{H}^{i}\left(G\left(\mathbb{F}_{q}\right), V\right)$ via $G$-cohomology of "translated" modules for $0 \leq i \leq s$ and $p \geq(2 s+1)(h-1)$. As a consequence of this formula, we prove in Corollary 4.2(B) that for $p \geq 3(h-1)$, if $\lambda$ is $G_{1}$-linked to the zero weight, then $\mathrm{H}^{1}\left(G\left(\mathbb{F}_{q}\right), L(\lambda)\right) \cong \mathrm{H}^{1}(G, L(\lambda))$ and if $\lambda$ is $p$-singular, then $\mathrm{H}^{1}\left(G\left(\mathbb{F}_{q}\right), L(\lambda)\right)=0$. Furthermore, we provide necessary conditions on $\lambda$ when $\lambda$ is not $G_{1}$-linked to the zero weight to have $\mathrm{H}^{1}\left(G\left(\mathbb{F}_{q}\right), L(\lambda)\right) \neq 0$ (see Proposition 4.6) and show that such weights exist (see Theorem 4.7). For $p \geq$ $3(h-1)$, Theorem 4.4 also provides a complete characterization of $\mathrm{H}^{1}\left(G\left(\mathbb{F}_{q}\right), H^{0}(\lambda)\right)$ where $H^{0}(\lambda)=\operatorname{ind}_{B}^{G} \lambda$. These results extend earlier calculations by Friedlander and Parshall $[\mathrm{F}],[\mathrm{FP}]$. Our methods also allow us to improve on results of Jantzen Jan6] and Andersen [And2] for large primes. This is accomplished by providing both necessary and sufficient conditions on the weights of the composition factors of a $G$-module $V$ (depending on the root system) to insure that the restriction map $\mathrm{H}^{1}(G, V) \rightarrow \mathrm{H}^{1}\left(G\left(\mathbb{F}_{q}\right), V\right)$ is an isomorphism. Finally, in Section 4.10, necessary conditions are provided to insure that $\mathrm{H}^{1}\left(G_{r}, L(\mu)\right) \cong \mathrm{H}^{1}(G, L(\mu))$.

In Section 5, we provide sufficient conditions on weights $\lambda, \mu \in X_{r}(T)$ to insure that the restriction maps

$$
\operatorname{Ext}_{G}^{1}(L(\lambda), L(\mu)) \rightarrow \operatorname{Ext}_{G\left(\mathbb{F}_{q}\right)}^{1}(L(\lambda), L(\mu))
$$

and

$$
\operatorname{Ext}_{G}^{1}(L(\lambda), L(\mu)) \rightarrow \operatorname{Ext}_{G_{r}}^{1}(L(\lambda), L(\mu))
$$

are isomorphisms (see Theorems 5.3, 5.6, and 5.7). Theorem 5.3(B), in particular, shows that the isomorphism holds if the weights $\lambda$ and $\mu$ are "close" together. This leads to several consequences presented in Section 5.4 involving extensions between modules whose highest weights lie in the same alcove or are mirror reflections across 
an alcove wall. As mentioned above, Theorem 5.3 of CPS2 shows that the Lusztig conjecture is equivalent to the nonvanishing of certain $G$-extensions between simple modules. Corollary $5.4(\mathrm{C})$ shows that some of these $G$-extensions can be identified with extensions over $G_{1}$.

Notation 1.2. The conventions in the paper will follow the ones used in Jan1. Throughout this paper, for the sake of clarity, we will assume that $G$ is a simple simply connected algebraic group defined and split over the finite field $\mathbb{F}_{p}$ with $p$ elements. We will work over the field $k$, where $k$ denotes the algebraic closure of $\mathbb{F}_{p}$. Many of our results can be extended easily to the case when $G$ is semisimple or reductive. Let $T$ be a maximal split torus in $G$ and $\Phi$ the associated root system. We will denote the positive roots with respect to a base of simple roots $\Delta$ by $\Phi^{+}$. The negative roots will be denoted by $\Phi^{-}$. Let $B$ be a Borel subgroup containing $T$ with respect to the negative roots and $U$ the unipotent radical of $B$.

Let $X(T)$ be the integral weight lattice obtained from $\Phi$ contained in the Euclidean space $\mathbb{E}$ with the inner product denoted by $\langle$,$\rangle . The set X(T)$ has a partial ordering given by $\lambda \geq \mu$ if and only if $\lambda-\mu \in \sum_{\alpha \in \Delta} \mathbb{N} \alpha$ for $\lambda, \mu \in X(T)$. Let $\alpha^{\vee}=2 \alpha /\langle\alpha, \alpha\rangle$ be the coroot corresponding to $\alpha \in \Phi$. The set of dominant integral weights is defined by

$$
X(T)_{+}=\left\{\lambda \in X(T): 0 \leq\left\langle\lambda, \alpha^{\vee}\right\rangle \text { for all } \alpha \in \Delta\right\}
$$

Furthermore, the set of $p^{r}$-restricted weights is

$$
X_{r}(T)=\left\{\lambda \in X(T): 0 \leq\left\langle\lambda, \alpha^{\vee}\right\rangle<p^{r} \text { for all } \alpha \in \Delta\right\} .
$$

The closure of the bottom $p$ alcove in $X(T)$ is given by

$$
\bar{C}_{\mathbb{Z}}=\left\{\lambda \in X(T): 0 \leq\left\langle\lambda+\rho, \alpha^{\vee}\right\rangle \leq p \text { for all } \alpha \in \Phi^{+}\right\} .
$$

The Weyl group $W$ is the group generated by the reflections $s_{\alpha}: \mathbb{E} \rightarrow \mathbb{E}$ given by $s_{\alpha}(\lambda)=\lambda-\left\langle\lambda, \alpha^{\vee}\right\rangle \alpha$. The group $W$ acts on $X(T)$ via the "dot action" given by $w \cdot \lambda=w(\lambda+\rho)-\rho$ where $\rho$ is the half sum of positive roots. The long word in $W$ will be denoted by $w_{0}$ and the Coxeter number for $\Phi$ is $h=\left\langle\rho, \alpha_{0}^{\vee}\right\rangle+1$, where $\alpha_{0}$ is the maximal short root.

For each $\lambda \in X(T)_{+}$, let $H^{0}(\lambda)=\operatorname{ind}_{B}^{G} \lambda$. The simple modules for $G$ are labeled by the set $X(T)_{+}$and are given by the correspondence $\lambda \rightarrow L(\lambda)=\operatorname{soc}_{G} H^{0}(\lambda)$. A complete set of nonisomorphic simple $G_{r}$-modules and simple $G\left(\mathbb{F}_{q}\right)$-modules are obtained by taking $\left\{L(\lambda): \lambda \in X_{r}(T)\right\}$.

Finally, we make use of the following sets of weights and modules, which were used in [BNP1, BNP2]. For each $s$ with $0 \leq s \leq p-1$, let

$$
\pi_{s, r}=\left\{\lambda \in X(T)_{+}:\left\langle\lambda+\rho, \alpha_{0}^{\vee}\right\rangle<2 s p^{r}\left\langle\rho, \alpha_{0}^{\vee}\right\rangle\right\}
$$

The set of weights $\pi_{s, r}$ is saturated. Further, let $\mathcal{C}_{s, r}$ be the full subcategory of $G$ modules all of whose composition factors have highest weights in $\pi_{s, r}$. For $s=1$, the category $\mathcal{C}_{1, r}$ essentially coincides with the $p^{r}$-bounded category as defined in [Jan1. p. 360]. Observe that if $\lambda \in \pi_{s, r}$ is expressed as $\lambda=\lambda^{\prime}+p^{r} \nu$ for weights $\lambda^{\prime} \in X_{r}(T)$ and $\nu \in X(T)_{+}$, then the weight $\nu$ satisfies $\left\langle\nu, \alpha_{0}^{\vee}\right\rangle\left\langle 2 s\left\langle\rho, \alpha_{0}^{\vee}\right\rangle=2 s(h-1)\right.$. This leads us to define, for a positive integer $m$, the set

$$
\Gamma_{m}=\left\{\nu \in X(T)_{+}:\left\langle\nu, \alpha_{0}^{\vee}\right\rangle<m\right\} .
$$

For convenience, we denote $\Gamma_{h}$ simply by $\Gamma$. 


\section{ACKNOWLEDGMENTS}

The first author thanks the Department of Mathematics and Statistics at the University of South Alabama and the Department of Mathematics and Statistics at Utah State University for their hospitality during the preparation of this paper.

\section{Summary of EXT' ${ }^{1}$-Formulas}

2.1. Let $\lambda \in X_{r}(T)$ and $\lambda=\sum_{i=0}^{r-1} p^{i} \lambda_{i}$ where $\lambda_{i} \in X_{1}(T)$. Set $\dot{\lambda}=\sum_{i=0}^{r-2} p^{i} \lambda_{i}$ so that $\lambda=\dot{\lambda}+p^{r-1} \lambda_{r-1}$. The following result summarizes a series of $\operatorname{Ext}^{1}$-formulas proved in [BNP2].

Theorem. Let $G$ be a simple simply connected algebraic group with $p \geq 3(h-1)$ and let $\lambda, \mu \in X_{r}(T)$. Then

(a) $\operatorname{Ext}_{G\left(\mathbb{F}_{q}\right)}^{1}(L(\lambda), L(\mu)) \cong \bigoplus_{\nu \in \Gamma} \operatorname{Ext}_{G}^{1}\left(L(\lambda) \otimes L(\nu)^{(r)}, L(\mu) \otimes L(\nu)\right)$.

(b) For $r \geq 2, \operatorname{Ext}_{G\left(\mathbb{F}_{q}\right)}^{1}(L(\lambda), L(\mu)) \cong \operatorname{Ext}_{G}^{1}(L(\lambda), L(\mu)) \oplus R$ where

$$
R=\bigoplus_{\nu \in \Gamma-\{0\}} \operatorname{Ext}_{G}^{1}\left(L\left(\lambda_{r-1}\right) \otimes L(\nu)^{(1)}, L\left(\mu_{r-1}\right)\right) \otimes \operatorname{Hom}_{G}(L(\dot{\lambda}), L(\dot{\mu}) \otimes L(\nu)) .
$$

Equivalently, in this case, we have

$$
\begin{gathered}
R=\bigoplus_{\nu \in \Gamma-\{0\}} \operatorname{Hom}_{G}\left(L(\nu), \operatorname{Ext}_{G_{1}}^{1}\left(L\left(\lambda_{r-1}\right), L\left(\mu_{r-1}\right)\right)^{(-1)}\right) \\
\otimes \operatorname{Hom}_{G}(L(\dot{\lambda}), L(\dot{\mu}) \otimes L(\nu)) .
\end{gathered}
$$

(c) For $r=1$, $\operatorname{Ext}_{G\left(\mathbb{F}_{p}\right)}^{1}(L(\lambda), L(\mu)) \cong \operatorname{Ext}_{G}^{1}(L(\lambda), L(\mu)) \oplus R$ where

$$
R=\bigoplus_{\nu \in \Gamma-\{0\}} \operatorname{Ext}_{G}^{1}\left(L(\lambda) \otimes L(\nu)^{(1)}, L(\mu) \otimes L(\nu)\right) .
$$

Equivalently, in this case, we have

$$
R=\bigoplus_{\nu \in \Gamma-\{0\}} \operatorname{Hom}_{G}\left(L(\nu)^{(1)}, \operatorname{Ext}_{G_{1}}^{1}(L(\lambda), L(\mu) \otimes L(\nu))\right) .
$$

2.2. The Ext ${ }^{1}$-result for simple $G$-modules given below was proved by Andersen And1, 5.6].

Theorem. Let $G$ be a simple simply connected algebraic group with $p \geq$ $3(h-1), r \geq 2$, and $\lambda, \mu \in X_{r}(T)$. Set $n=\min \left(\left\{i \mid \lambda_{i} \neq \mu_{i}\right\} \cup\{r-1\}\right)$ and $\lambda^{\prime}=\sum_{i=n+1}^{r-1} p^{i-n-1} \lambda_{i}, \mu^{\prime}=\sum_{i=n+1}^{r-1} p^{i-n-1} \mu_{i}$. Then

$\operatorname{Ext}_{G}^{1}(L(\lambda), L(\mu)) \cong \bigoplus_{\nu \in \Gamma} \operatorname{Ext}_{G}^{1}\left(L\left(\lambda_{n}\right) \otimes L(\nu)^{(1)}, L\left(\mu_{n}\right)\right) \otimes \operatorname{Hom}_{G}\left(L\left(\lambda^{\prime}\right), L\left(\mu^{\prime}\right) \otimes L(\nu)\right)$.

The preceding result has been stated in general for completeness. The reader will note that if $n=r-1$, then $\lambda^{\prime}=0=\mu^{\prime}$ and the conclusion reduces to the fact that

$$
\operatorname{Ext}_{G}^{1}(L(\lambda), L(\mu)) \cong \operatorname{Ext}_{G}^{1}\left(L\left(\lambda_{r-1}\right), L\left(\mu_{r-1}\right)\right),
$$

which follows readily from the Lyndon-Hochschild-Serre spectral sequence for $G_{r-1} \subset G$. Further, if $\lambda=\mu$, these Ext-groups are zero. 
2.3. We will now prove a result which will allow us to refine the Ext ${ }^{1}$-formulas given in Sections 2.1 and 2.2. The following proposition has previously been stated for special cases (rank two groups and fundamental weights $\nu$ ) in [Ye1 Prop. 4.4], [Ye2], and [Yeh].

Proposition. Let $G$ be a simple simply connected algebraic group with $p \geq 2(h-1)$, $\lambda_{0}, \mu_{0} \in X_{1}(T)$, and $\lambda_{1}, \mu_{1} \in X(T)_{+}$. Suppose that $\nu \in X(T)_{+}$with $\left\langle\nu, \alpha_{0}^{\vee}\right\rangle<p$. Then

$$
\begin{aligned}
& \operatorname{Hom}_{G}\left(L\left(\lambda_{0}+p \lambda_{1}\right), L\left(\mu_{0}+p \mu_{1}\right) \otimes L(\nu)\right) \\
& \quad \cong \operatorname{Hom}_{G}\left(L\left(\lambda_{0}\right), L\left(\mu_{0}\right) \otimes L(\nu)\right) \otimes \operatorname{Hom}_{G}\left(L\left(\lambda_{1}\right), L\left(\mu_{1}\right)\right) .
\end{aligned}
$$

In particular, $\operatorname{Hom}_{G}\left(L\left(\lambda_{0}+p \lambda_{1}\right), L\left(\mu_{0}+p \mu_{1}\right) \otimes L(\nu)\right)=0$, unless $\lambda_{1}=\mu_{1}$.

Proof. Let $M=\operatorname{Hom}_{G}\left(L\left(\lambda_{0}+p \lambda_{1}\right), L\left(\mu_{0}+p \mu_{1}\right) \otimes L(\nu)\right)$. According to BNP2] Prop. 2.3],

$$
\begin{aligned}
M & \cong\left(\operatorname{Hom}_{G_{1}}\left(L\left(\lambda_{0}+p \lambda_{1}\right), L\left(\mu_{0}+p \mu_{1}\right) \otimes L(\nu)\right)\right)^{G / G_{1}} \\
& \cong\left(\operatorname{Hom}_{G_{1}}\left(L\left(\lambda_{0}\right), L\left(\mu_{0}\right) \otimes L(\nu)\right) \otimes L\left(-w_{0} \lambda_{1}\right)^{(1)} \otimes L\left(\mu_{1}\right)^{(1)}\right)^{G / G_{1}} \\
& \cong\left(\operatorname{Hom}_{G}\left(L\left(\lambda_{0}\right), L\left(\mu_{0}\right) \otimes L(\nu)\right) \otimes L\left(-w_{0} \lambda_{1}\right)^{(1)} \otimes L\left(\mu_{1}\right)^{(1)}\right)^{G / G_{1}} \\
& \cong \operatorname{Hom}_{G}\left(L\left(\lambda_{0}\right), L\left(\mu_{0}\right) \otimes L(\nu)\right) \otimes\left(L\left(-w_{0} \lambda_{1}\right)^{(1)} \otimes L\left(\mu_{1}\right)^{(1)}\right)^{G / G_{1}} \\
& \cong \operatorname{Hom}_{G}\left(L\left(\lambda_{0}\right), L\left(\mu_{0}\right) \otimes L(\nu)\right) \otimes \operatorname{Hom}_{G}\left(L\left(\lambda_{1}\right), L\left(\mu_{1}\right)\right) .
\end{aligned}
$$

2.4. For $\sigma, \delta \in X(T)_{+}$, let $S(\sigma, \delta)=\operatorname{Hom}_{G}(L(\sigma), L(\delta))$. Also, for $r \geq 3$, if $\lambda \in X_{r}(T)$ and $\lambda=\sum_{i=0}^{r-1} p^{i} \lambda_{i}$ where $\lambda_{i} \in X_{1}(T)$, set $\ddot{\lambda}=\sum_{i=1}^{r-2} p^{i-1} \lambda_{i}$ and for $r=2$ set $\ddot{\lambda}=0$. For $r \geq 2$, the following theorem refines the results in Sections 2.1 and 2.2 .

Theorem. Let $G$ be a simple simply connected algebraic group. Moreover, let $p \geq 3(h-1), r \geq 2$, and $\lambda, \mu \in X_{r}(T)$. Set $n=\min \left(\left\{i \mid \lambda_{i} \neq \mu_{i}\right\} \cup\{r-1\}\right)$ and $\lambda_{r}=0=\mu_{r}$. If $0 \leq n<r-2$, set $\lambda^{\prime \prime}=\sum_{i=n+2}^{r-1} p^{i-n-2} \lambda_{i}$ and $\mu^{\prime \prime}=$ $\sum_{i=n+2}^{r-1} p^{i-n-2} \mu_{i}$. If $n=r-2$ or $r-1$, set $\lambda^{\prime \prime}=0=\mu^{\prime \prime}$. Then

(a) $\operatorname{Ext}_{G}^{1}(L(\lambda), L(\mu)) \cong \bigoplus_{\nu \in \Gamma} \operatorname{Ext}_{G}^{1}\left(L\left(\lambda_{n}\right) \otimes L(\nu)^{(1)}, L\left(\mu_{n}\right)\right) \otimes \operatorname{Hom}_{G}\left(L\left(\lambda_{n+1}\right)\right.$, $\left.L\left(\mu_{n+1}\right) \otimes L(\nu)\right) \otimes S\left(\lambda^{\prime \prime}, \mu^{\prime \prime}\right)$.

(b) $\operatorname{Ext}_{G\left(\mathbb{F}_{q}\right)}^{1}(L(\lambda), L(\mu)) \cong \operatorname{Ext}_{G}^{1}(L(\lambda), L(\mu)) \oplus R$ where

$$
\begin{aligned}
& R=\bigoplus_{\nu \in \Gamma-\{0\}} \operatorname{Ext}_{G}^{1}\left(L\left(\lambda_{r-1}\right) \otimes L(\nu)^{(1)}, L\left(\mu_{r-1}\right)\right) \\
& \otimes \operatorname{Hom}_{G}\left(L\left(\lambda_{0}\right), L\left(\mu_{0}\right) \otimes L(\nu)\right) \otimes S(\ddot{\lambda}, \ddot{\mu}) .
\end{aligned}
$$

Proof. (a) Consider $\lambda^{\prime}$ and $\mu^{\prime}$ as defined in Theorem 2.2. Since $\lambda^{\prime}=\lambda_{n+1}+p \lambda^{\prime \prime}$ and $\mu^{\prime}=\mu_{n+1}+p \mu^{\prime \prime}$, part (a) follows from Theorem 2.2 and Proposition 2.3. (b) Similarly, with $\dot{\lambda}$ and $\dot{\mu}$ as defined in Section 2.1, we have $\dot{\lambda}=\lambda_{0}+p \ddot{\lambda}$ and $\dot{\mu}=\mu_{0}+p \ddot{\mu}$. So, the claim follows from Theorem 2.1(b) and Proposition 2.3.

In [AJL, 3.9, 4.5] it was observed that extensions between two simple modules for the algebraic group of type $A_{1}$ and the corresponding finite groups vanish unless the $p$-adic expansions of the weights differ in at most 2 consecutive slots. We can generalize this observation to groups of other types, provided the prime is sufficiently large. 
Corollary. Let $G$ be a simple simply connected algebraic group. Moreover, let $p \geq 3(h-1), r \geq 2$, and $\lambda, \mu \in X_{r}(T)$.

(a) $\operatorname{Ext}_{G}^{1}(L(\lambda), L(\mu))=0$ unless there exists an $n$ with $0 \leq n \leq r-1$ such that $\lambda_{n} \neq \mu_{n}$ and $\lambda_{i}=\mu_{i}$ for all $i \neq n, n+1$.

(b) $\operatorname{Ext}_{G\left(\mathbb{F}_{q}\right)}^{1}(L(\lambda), L(\mu))=0$ unless there exists an $n$ with $0 \leq n \leq r-1$ such that $\lambda_{n} \neq \mu_{n}$ and $\lambda_{i}=\mu_{i}$ for all $i \neq \equiv n, n+1(\bmod r)$.

Proof. Since $\operatorname{Ext}_{G}^{1}(L(\lambda), L(\lambda))=0$ and $\operatorname{Ext}_{G\left(\mathbb{F}_{q}\right)}^{1}(L(\lambda), L(\lambda))=0$ (by [BNP2, 3.4]), in either case, there is some $n$ with $0 \leq n \leq r-1$ for which $\lambda_{n} \neq \mu_{n}$. Note that when $r=2$, this is all that is claimed and so we may assume that $r \geq 3$. For part (a), let $n$ be the least nonnegative integer with $\lambda_{n} \neq \mu_{n}$. By part (a) of the preceding theorem, $\operatorname{Ext}_{G}^{1}(L(\lambda), L(\mu))=0$ unless $\lambda^{\prime \prime}=\mu^{\prime \prime}$. In other words, if the Ext-group is nonzero, we must have $\lambda_{i}=\mu_{i}$ for all $i \neq n, n+1$.

For part (b), suppose there is an $i$ with $1 \leq i \leq r-2$ and $\lambda_{i} \neq \mu_{i}$. Then $\ddot{\lambda} \neq \ddot{\mu}$ and by part (b) of the preceding theorem, $\operatorname{Ext}_{G\left(\mathbb{F}_{q}\right)}^{1}(L(\lambda), L(\mu)) \cong \operatorname{Ext}_{G}^{1}(L(\lambda), L(\mu))$. Hence, by part (a), for these groups to be nonzero, we must have $\lambda_{n} \neq \mu_{n}$ for some (minimal) $n$ and $\lambda_{i}=\mu_{i}$ for $i \neq n, n+1$. Alternately, for $\operatorname{Ext}_{G\left(\mathbb{F}_{q}\right)}^{1}(L(\lambda), L(\mu))$ to possibly be nonzero, we must have $\lambda_{i}=\mu_{i}$ for all $1 \leq i \leq r-2$.

2.5. It is easy to see from Theorem 2.4(a) that Ye's results, [Ye1 Thm. 5.1] and [Ye2, Thm. 5], for extensions of simple modules for rank 2 groups can be generalized as follows. We use the same notation as in Ye2 and define for $\lambda \in X(T)_{+}$,

$$
A(\lambda)=\left\{\mu \in X(T)_{+} \mid \operatorname{Ext}_{G}^{1}(L(\lambda), L(\mu)) \neq 0\right\}
$$

and for $\lambda_{0} \in X_{1}(T)$,

$$
B\left(\lambda_{0}\right)=\left\{\gamma \in \pi_{1,1} \mid \operatorname{Ext}_{G}^{1}\left(L\left(\lambda_{0}\right), L(\gamma)\right) \neq 0\right\} .
$$

Theorem. Let $G$ be a simple simply connected algebraic group with $p \geq 3(h-1)$. For $\lambda=\lambda_{0}+p \lambda_{1}+p^{2} \bar{\lambda}$ with $\lambda_{0}, \lambda_{1} \in X_{1}(T), \bar{\lambda} \in X(T)_{+}$, one has

$$
\begin{aligned}
A(\lambda)=\left\{\lambda_{0}+p A\left(\lambda_{1}+p \bar{\lambda}\right)\right\} \cup\{ & \mu_{0}+p \mu_{1}+p^{2} \bar{\lambda} \mid \mu_{0}+p \nu \in B\left(\lambda_{0}\right), L\left(\mu_{1}\right) \\
& \left.\subset \operatorname{soc}_{G}\left(L\left(\lambda_{1}\right) \otimes L(\nu)\right), \mu_{0}, \mu_{1} \in X_{1}(T), \nu \in \Gamma\right\} .
\end{aligned}
$$

2.6. A decomposition similar to that of Theorem 2.1 for extensions over finite Chevalley groups can also be obtained for extensions over Frobenius kernels $G_{r}$.

Theorem. Let $G$ be a simple simply connected algebraic group with $p \geq 3(h-1)$ and $\lambda, \mu \in X_{r}(T)$. Then

$$
\operatorname{Ext}_{G_{r}}^{1}(L(\lambda), L(\mu)) \cong \operatorname{Ext}_{G}^{1}(L(\lambda), L(\mu)) \oplus R,
$$

where

$$
R=\bigoplus_{\nu \in \Gamma-\{0\}} L(\nu)^{(r)} \otimes \operatorname{Ext}_{G}^{1}\left(L(\lambda) \otimes L(\nu)^{(r)}, L(\mu)\right) .
$$

Proof. It follows immediately from [And1, Proposition 5.5] that

$$
\operatorname{Ext}_{G_{r}}^{1}(L(\lambda), L(\mu)) \cong \bigoplus_{\nu \in \Gamma_{2 h-1}} L(\nu)^{(r)} \otimes \operatorname{Ext}_{G}^{1}\left(L(\lambda) \otimes L(\nu)^{(r)}, L(\mu)\right) .
$$

Moreover, if $\nu \in X(T)_{+}$satisfies $\left\langle\nu, \alpha_{0}^{\vee}\right\rangle<2(h-1)$ and $\operatorname{Ext}_{G}^{1}\left(L(\lambda) \otimes L(\nu)^{(r)}, L(\mu)\right) \neq$ 0 , then by [BNP2, Prop. 2.4], $\nu$ must lie in $\Gamma$. 
2.7. The results of Section 2.4 can be used to simplify the decomposition in Theorem 2.6 by reducing computations for $p^{r}$-restricted weights to computations involving $p$-restricted weights. Two reduction lemmas are required for this. The first one states that when weights $\lambda$ and $\mu$ have a common term in their $p$-adic expansions, this term can often be "deleted" and the $G$-extensions remain the same. The isomorphism follows readily from Theorem 2.4(a) by comparing the two direct sum decompositions (one for each Ext-group) and observing that they are the same.

Lemma (A). Let $G$ be a simple simply connected algebraic group with $p \geq 3(h-1)$ and $\lambda, \mu \in X(T)_{+}$. Let $m \geq 0$ be an integer such that $\lambda_{m}=\mu_{m}$. For $m>0$, suppose also that $m-1$ is not the least integer $n$ with $\lambda_{n} \neq \mu_{n}$. Set $\bar{\lambda}=\sum_{i=0}^{m-1} p^{i} \lambda_{i}+$ $\sum_{i=m+1}^{\infty} p^{i-1} \lambda_{i}$ and $\bar{\mu}=\sum_{i=0}^{m-1} p^{i} \mu_{i}+\sum_{i=m+1}^{\infty} p^{i-1} \mu_{i}$. Then $\operatorname{Ext}_{G}^{1}(L(\lambda), L(\mu)) \cong$ $\operatorname{Ext}_{G}^{1}(L(\bar{\lambda}), L(\bar{\mu}))$.

Lemma (B). Let $G$ be a simple simply connected algebraic group with $p \geq 3(h-1)$, $r \geq 2$, and $\lambda, \mu \in X_{r}(T)$. Then for $\nu \in \Gamma-\{0\}$,

$$
\operatorname{Ext}_{G}^{1}\left(L(\lambda) \otimes L(\nu)^{(r)}, L(\mu)\right) \cong \begin{cases}0 & \text { if } \dot{\lambda} \neq \dot{\mu}, \\ \operatorname{Ext}_{G}^{1}\left(L\left(\lambda_{r-1}\right) \otimes L(\nu)^{(1)}, L\left(\mu_{r-1}\right)\right) & \text { if } \dot{\lambda}=\dot{\mu} .\end{cases}
$$

Proof. It follows immediately from Corollary 2.4(a) (applied to $\lambda+p^{r} \nu$ and $\mu$ ) that the Ext-group is zero unless $\dot{\lambda}=\dot{\mu}$. In that case, the isomorphism follows by repeated application of Lemma $2.7(\mathrm{~A})$.

When $r \geq 2$, Lemma 2.7(B) immediately shows that Theorem 2.6 can be improved so that the remainder term involves $G$-extensions over only $p$-restricted weights. In the case that $r=1$, the following is simply a restatement of Theorem 2.6 .

Theorem. Let $G$ be a simple simply connected algebraic group with $p \geq 3(h-1)$ and $\lambda, \mu \in X_{r}(T)$. Then

$$
\operatorname{Ext}_{G_{r}}^{1}(L(\lambda), L(\mu)) \cong \operatorname{Ext}_{G}^{1}(L(\lambda), L(\mu)) \oplus R
$$

where

$$
R= \begin{cases}0 & \text { if } \dot{\lambda} \neq \dot{\mu}, \\ \bigoplus_{\nu \in \Gamma-\{0\}} L(\nu)^{(r)} \otimes \operatorname{Ext}_{G}^{1}\left(L\left(\lambda_{r-1}\right) \otimes L(\nu)^{(1)}, L\left(\mu_{r-1}\right)\right) & \text { if } \dot{\lambda}=\dot{\mu} .\end{cases}
$$

Corollary (A). Let $G$ be a simple simply connected algebraic group with $p \geq$ $3(h-1)$ and $\lambda, \mu \in X_{r}(T)$. Then

$$
\operatorname{Ext}_{G_{r}}^{1}(L(\lambda), L(\mu)) \cong \begin{cases}\operatorname{Ext}_{G}^{1}(L(\lambda), L(\mu)) & \text { if } \dot{\lambda} \neq \dot{\mu} \\ \operatorname{Ext}_{G_{1}}^{1}\left(L\left(\lambda_{r-1}\right), L\left(\mu_{r-1}\right)\right)^{(r-1)} & \text { if } \dot{\lambda}=\dot{\mu} .\end{cases}
$$

Proof. The case $\dot{\lambda} \neq \dot{\mu}$ follows immediately from the theorem. The latter case follows from the theorem by comparing the decomposition for the two Ext-groups. Alternately, it follows from the Lyndon-Hochschild-Serre spectral sequence for $G_{1} \unlhd$ $G_{r}$.

Note that in the latter case of the corollary, these groups may or may not be isomorphic to $\operatorname{Ext}_{G}^{1}(L(\lambda), L(\mu))$. Finally, we note the following immediate consequence of the theorem. 
Corollary (B). Let $G$ be a simple simply connected algebraic group with $p \geq$ $3(h-1)$ and $r \geq 2$. Suppose $\lambda, \mu \in X_{r-1}(T)$. Then

$$
\operatorname{Ext}_{G_{r}}^{1}(L(\lambda), L(\mu)) \cong \begin{cases}\operatorname{Ext}_{G}^{1}(L(\lambda), L(\mu)) & \text { if } \lambda \neq \mu, \\ 0 & \text { if } \lambda=\mu .\end{cases}
$$

\section{COMPARISON RESUltS FOR Finite GROUPS: $r \geq 2$}

3.1. The results of Section 2 allow one to compute extensions of simple $G\left(\mathbb{F}_{q}\right)$ modules by computing certain families of extensions over $G$. The goal of this section is to identify the extensions between a pair of simple $G\left(\mathbb{F}_{q}\right)$-modules with the $G$ extensions of a single pair of simple $G$-modules. The following theorem presents one approach to this problem. Note that the integer $n$ defined here is different from that used in Theorems 2.2 and 2.4. Further, note that if $n=r-1$, then the weights $\widetilde{\lambda}$ and $\widetilde{\mu}$ defined below simply equal $\lambda$ and $\mu$ respectively.

Theorem. Let $G$ be a simple simply connected algebraic group with $p \geq 3(h-1)$ and $\lambda, \mu \in X_{r}(T)$. Set $n=\min \left(\left\{i \mid \lambda_{i}=\mu_{i}\right\} \cup\{r-1\}\right)$ and define $p^{r}$-restricted weights $\widetilde{\lambda}=\sum_{i=n+1}^{n+r} p^{i-n-1} \lambda_{i}$ and $\widetilde{\mu}=\sum_{i=n+1}^{n+r} p^{i-n-1} \mu_{i}$, where $\lambda_{r+i}=\lambda_{i}$ and $\mu_{r+i}=\mu_{i}$.

(a) If $r \geq 3$, then

$$
\operatorname{Ext}_{G\left(\mathbb{F}_{q}\right)}^{1}(L(\lambda), L(\mu)) \cong \operatorname{Ext}_{G}^{1}(L(\widetilde{\lambda}), L(\widetilde{\mu})) .
$$

(b) If $r=2$ and $\lambda_{0}=\mu_{0}$ or $\lambda_{1}=\mu_{1}$, then

$$
\operatorname{Ext}_{G\left(\mathbb{F}_{q}\right)}^{1}(L(\lambda), L(\mu)) \cong \operatorname{Ext}_{G}^{1}(L(\widetilde{\lambda}), L(\widetilde{\mu})) .
$$

(c) If $r=2$ and $\lambda_{i} \neq \mu_{i}$ for $i=0,1$, set $\hat{\lambda}=\lambda_{1}+p \lambda_{0}$ and $\hat{\mu}=\mu_{1}+p \mu_{0}$. Then

$$
\operatorname{Ext}_{G\left(\mathbb{F}_{q}\right)}^{1}(L(\lambda), L(\mu)) \cong \operatorname{Ext}_{G}^{1}(L(\lambda), L(\mu)) \oplus \operatorname{Ext}_{G}^{1}(L(\hat{\lambda}), L(\hat{\mu})) \text {. }
$$

Proof. Since the Frobenius map is an automorphism of $G\left(\mathbb{F}_{q}\right)$, for any finitedimensional $G$-module $V$ and any positive integer $m$,

$$
H^{1}\left(G\left(\mathbb{F}_{q}\right), V\right) \cong H^{1}\left(G\left(\mathbb{F}_{q}\right), V^{(m)}\right) \text {. }
$$

Therefore,

$$
\operatorname{Ext}_{G\left(\mathbb{F}_{q}\right)}^{1}(L(\lambda), L(\mu)) \cong \operatorname{Ext}_{G\left(\mathbb{F}_{q}\right)}^{1}\left(L(\lambda)^{(m)}, L(\mu)^{(m)}\right) .
$$

For $n=\min \left(\left\{i \mid \lambda_{i}=\mu_{i}\right\} \cup\{r-1\}\right), L(\widetilde{\lambda}) \cong L(\lambda)^{(r-1-n)}$ and $L(\widetilde{\mu}) \cong L(\mu)^{(r-1-n)}$ as $G\left(\mathbb{F}_{q}\right)$-modules. Hence, we may identify

$$
\operatorname{Ext}_{G\left(\mathbb{F}_{q}\right)}^{1}(L(\lambda), L(\mu)) \cong \operatorname{Ext}_{G\left(\mathbb{F}_{q}\right)}^{1}(L(\widetilde{\lambda}), L(\widetilde{\mu})) .
$$

(a) For $r \geq 3$, Corollary 2.4 implies that

$$
\operatorname{Ext}_{G\left(\mathbb{F}_{q}\right)}^{1}(L(\lambda), L(\mu))=0 \text { and } \operatorname{Ext}_{G}^{1}(L(\widetilde{\lambda}), L(\widetilde{\mu}))=0
$$

unless there exists an $i$ with $0 \leq i \leq r-1$ and $\lambda_{i}=\mu_{i}$. Suppose such an $i$ exists and apply Theorem 2.1(b) to $\widetilde{\lambda}$ and $\widetilde{\mu}$. Since $\widetilde{\lambda}_{r-1}=\widetilde{\mu}_{r-1}, \operatorname{Ext}_{G_{1}}^{1}\left(L\left(\widetilde{\lambda}_{r-1}\right), L\left(\widetilde{\mu}_{r-1}\right)\right)=0$ by [And1 and so the remainder term $R$ is zero. Hence, it follows that

$$
\operatorname{Ext}_{G\left(\mathbb{F}_{q}\right)}^{1}(L(\lambda), L(\mu)) \cong \operatorname{Ext}_{G\left(\mathbb{F}_{q}\right)}^{1}(L(\widetilde{\lambda}), L(\widetilde{\mu})) \cong \operatorname{Ext}_{G}^{1}(L(\widetilde{\lambda}), L(\widetilde{\mu}))
$$

and part (a) holds.

(b) For $r=2$, if $\lambda_{0}=\mu_{0}$ or $\lambda_{1}=\mu_{1}$, then $\widetilde{\lambda}_{r-1}=\widetilde{\mu}_{r-1}$ and the claim follows by the same argument as for $r \geq 3$. 
(c) Suppose $r=2$ and $\lambda_{i} \neq \mu_{i}$ for $i=0,1$. By Theorem 2.1(b), we have

$$
\operatorname{Ext}_{G\left(\mathbb{F}_{q}\right)}^{1}(L(\lambda), L(\mu)) \cong \operatorname{Ext}_{G}^{1}(L(\lambda), L(\mu)) \oplus R
$$

where

$$
R=\bigoplus_{\nu \in \Gamma-\{0\}} \operatorname{Ext}_{G}^{1}\left(L\left(\lambda_{1}\right) \otimes L(\nu)^{(1)}, L\left(\mu_{1}\right)\right) \otimes \operatorname{Hom}_{G}\left(L\left(\lambda_{0}\right), L\left(\mu_{0}\right) \otimes L(\nu)\right) .
$$

Since $\lambda_{0} \neq \mu_{0}$, we have

$$
R=\bigoplus_{\nu \in \Gamma} \operatorname{Ext}_{G}^{1}\left(L\left(\lambda_{1}\right) \otimes L(\nu)^{(1)}, L\left(\mu_{1}\right)\right) \otimes \operatorname{Hom}_{G}\left(L\left(\lambda_{0}\right), L\left(\mu_{0}\right) \otimes L(\nu)\right) .
$$

By Theorem 2.4(a), the remainder term $R$ is exactly $\operatorname{Ext}_{G}^{1}(L(\hat{\lambda}), L(\hat{\mu}))$.

3.2. For $r \geq 2$ and $p \geq 3(h-1)$, Theorem 3.1 provides a surprisingly simple answer to the following question raised by Humphreys [Hum] and Andersen [And2]: can every extension between two simple modules $L(\lambda)$ and $L(\mu)$ for the finite group $G\left(\mathbb{F}_{q}\right)$ be found via the algebraic group $G$ ?

We give an explicit construction of any nontrivial extension of $L(\lambda)$ by $L(\mu)$ for $G\left(\mathbb{F}_{q}\right)$. For a given pair $\lambda, \mu$, consider the integer $n$ and weights $\tilde{\lambda}, \tilde{\mu}$ described in Theorem 3.1. Let $E$ denote a nontrivial extension of $L(\widetilde{\lambda})$ by $L(\widetilde{\mu})$ for $G$. We twist $E n$ times with the Frobenius morphism and denote the result by $E^{(n)}$. A slight modification of the argument in [And2, Prop. 2.7] shows that $E^{(n)}$ remains nontrivial when restricted to $G\left(\mathbb{F}_{q}\right)$. Now $L(\widetilde{\lambda})^{(n)} \cong L(\lambda)$ and $L(\widetilde{\mu})^{(n)} \cong L(\mu)$ as $G\left(\mathbb{F}_{q}\right)$-modules. Hence, we constructed a nontrivial extension of $L(\lambda)$ by $L(\mu)$ for the finite group. It follows from Theorem 3.1 that all nontrivial extensions can be found in this fashion. Notice that in the case $r=2$ and $\lambda_{i} \neq \mu_{i}$ for $i=0,1$, one needs to consider, in addition, the weights $\hat{\lambda}$ and $\hat{\mu}$. But the construction remains the same. For $r \geq 3$, the preceding construction can be done with at most one Frobenius twist if a slightly different choice of the weights $\widetilde{\lambda}$ and $\widetilde{\mu}$ is made.

3.3. Let $\lambda, \mu \in X_{r}(T)$. In [AJL, Corollary 4.5 (b)], the authors showed that $\operatorname{dim}_{k} \operatorname{Ext}_{S L_{2}\left(\mathbb{F}_{q}\right)}^{1}(L(\lambda), L(\mu)) \leq 1$, unless $r=2$ and $\lambda_{1}=\mu_{0}=(p-1) / 2$ and $\lambda_{0}=\mu_{1}=(p-3) / 2$ or $\lambda_{1}=\mu_{0}=(p-3) / 2$ and $\lambda_{0}=\mu_{1}=(p-1) / 2$. In that case the dimension is 2 . The weights $(p-3) / 2$ and $(p-1) / 2$ are exactly the ones for which the finite group $S L_{2}\left(\mathbb{F}_{p}\right)$ has self-extensions. This suggests that there is a connection between the existence of self-extensions for $G\left(\mathbb{F}_{p}\right)$ and a "doubling" of the dimension for certain extensions for the group $G\left(\mathbb{F}_{p^{2}}\right)$. Using Theorem 3.1, we prove the following generalization for groups of higher rank. We use the notation $\widetilde{\lambda}$ and $\hat{\lambda}$ as defined in Theorem 3.1.

Theorem. Let $G$ be a simple simply connected algebraic group with $p \geq 3(h-1)$, $r \geq 2$, and $\lambda, \mu \in X_{r}(T)$. If $r \neq 2$ or the underlying root system is not of type $A_{1}$ or $C_{n}$, then

$$
\begin{aligned}
& \max \left\{\operatorname{dim}_{k} \operatorname{Ext}_{G\left(\mathbb{F}_{q}\right)}^{1}(L(\lambda), L(\mu)) \mid \lambda, \mu \in X_{r}(T)\right\} \\
& =\max \left\{\operatorname{dim}_{k} \operatorname{Ext}_{G}^{1}(L(\lambda), L(\mu)) \mid \lambda, \mu \in X_{r}(T)\right\} .
\end{aligned}
$$

Otherwise,

$$
\begin{aligned}
& \max \left\{\operatorname{dim}_{k} \operatorname{Ext}_{G\left(\mathbb{F}_{q}\right)}^{1}(L(\lambda), L(\mu)) \mid \lambda, \mu \in X_{r}(T)\right\} \\
& \leq 2 \max \left\{\operatorname{dim}_{k} \operatorname{Ext}_{G}^{1}(L(\lambda), L(\mu)) \mid \lambda, \mu \in X_{r}(T)\right\}
\end{aligned}
$$


Proof. By Theorem 3.1, $\operatorname{Ext}_{G\left(\mathbb{F}_{q}\right)}^{1}(L(\lambda), L(\mu)) \cong \operatorname{Ext}_{G}^{1}(L(\widetilde{\lambda}), L(\widetilde{\mu}))$ unless $r=2$, $\lambda_{0} \neq \mu_{0}$, and $\lambda_{1} \neq \mu_{1}$. In the remaining case, we have

$$
\operatorname{Ext}_{G\left(\mathbb{F}_{q}\right)}^{1}(L(\lambda), L(\mu)) \cong \operatorname{Ext}_{G}^{1}(L(\lambda), L(\mu)) \oplus \operatorname{Ext}_{G}^{1}(L(\hat{\lambda}), L(\hat{\mu})) .
$$

If $\operatorname{Ext}_{G\left(\mathbb{F}_{q}\right)}^{1}(L(\lambda), L(\mu)) \neq 0$, then the first dimension criterion fails only if both

$$
\operatorname{Ext}_{G}^{1}(L(\lambda), L(\mu)) \neq 0 \text { and } \operatorname{Ext}_{G}^{1}(L(\hat{\lambda}), L(\hat{\mu})) \neq 0 .
$$

Suppose these are both nonzero. By Theorem 2.4, there exists $\nu_{1}, \nu_{2} \in \Gamma$ such that

$$
\begin{gathered}
\operatorname{Ext}_{G}^{1}\left(L\left(\lambda_{0}\right) \otimes L\left(\nu_{1}\right)^{(1)}, L\left(\mu_{0}\right)\right) \neq 0, \\
\operatorname{Ext}_{G}^{1}\left(L\left(\lambda_{1}\right) \otimes L\left(\nu_{2}\right)^{(1)}, L\left(\mu_{1}\right)\right) \neq 0, \\
0 \neq \operatorname{Hom}_{G}\left(L\left(\lambda_{0}\right), L\left(\mu_{0}\right) \otimes L\left(\nu_{2}\right)\right) \cong \operatorname{Hom}_{G}\left(L\left(\mu_{0}\right), L\left(\lambda_{0}\right) \otimes L\left(-w_{0} \nu_{2}\right)\right),
\end{gathered}
$$

and

$$
0 \neq \operatorname{Hom}_{G}\left(L\left(\lambda_{1}\right), L\left(\mu_{1}\right) \otimes L\left(\nu_{1}\right)\right) \cong \operatorname{Hom}_{G}\left(L\left(\mu_{1}\right), L\left(\lambda_{1}\right) \otimes L\left(-w_{0} \nu_{1}\right)\right) .
$$

Since $\lambda_{0} \neq \mu_{0}$ and $\lambda_{1} \neq \mu_{1}$, both $\nu_{1}$ and $\nu_{2}$ are nonzero. Define the $G$-module $Q$ via the exact sequence

$$
0 \rightarrow L\left(\mu_{0}\right) \rightarrow L\left(\lambda_{0}\right) \otimes L\left(-w_{0} \nu_{2}\right) \rightarrow Q \rightarrow 0 .
$$

We look at the long exact Ext-sequence

$$
\begin{gathered}
\cdots \rightarrow \operatorname{Hom}_{G}\left(L\left(\lambda_{0}\right) \otimes L\left(\nu_{1}\right)^{(1)}, Q\right) \rightarrow \operatorname{Ext}_{G}^{1}\left(L\left(\lambda_{0}\right) \otimes L\left(\nu_{1}\right)^{(1)}, L\left(\mu_{0}\right)\right) \\
\rightarrow \operatorname{Ext}_{G}^{1}\left(L\left(\lambda_{0}\right) \otimes L\left(\nu_{1}\right)^{(1)}, L\left(\lambda_{0}\right) \otimes L\left(-w_{0} \nu_{2}\right)\right) \rightarrow \cdots
\end{gathered}
$$

The weights of $Q$ are less than $\lambda_{0}-w_{0} \nu_{2}$. Since $\nu_{1} \neq 0$, $\operatorname{Hom}_{G}\left(L\left(\lambda_{0}\right) \otimes L\left(\nu_{1}\right)^{(1)}, Q\right)=$ 0 and the map $\operatorname{Ext}_{G}^{1}\left(L\left(\lambda_{0}\right) \otimes L\left(\nu_{1}\right)^{(1)}, L\left(\mu_{0}\right)\right) \rightarrow \operatorname{Ext}_{G}^{1}\left(L\left(\lambda_{0}\right) \otimes L\left(\nu_{1}\right)^{(1)}, L\left(\lambda_{0}\right) \otimes\right.$ $\left.L\left(-w_{0} \nu_{2}\right)\right)$ is an embedding. Therefore, $\operatorname{Ext}_{G}^{1}\left(L\left(\lambda_{0}\right) \otimes L\left(\nu_{1}\right)^{(1)}, L\left(\lambda_{0}\right) \otimes L\left(-w_{0} \nu_{2}\right)\right)$ $\neq 0$. But by [BNP2, Cor. 4.9] that is not possible unless $\Phi$ is of type $A_{1}$ or $C_{n}$.

We have shown that the "doubling" of the dimension of the extensions for $G\left(\mathbb{F}_{q}\right)$ happens only for $q=p^{2}$ and groups of type $A_{1}$ and $C_{n}$. Moreover, a closer look at [BNP2, Cor. 4.9] shows that the $p$-adic expansion of weights for which such a doubling might occur are also candidates for self-extensions for $G\left(\mathbb{F}_{p}\right)$. In the following example, one can see that Humphreys' examples of self-extensions for the group $S p_{4}\left(\mathbb{F}_{p}\right)$ give rise to a doubling of the dimensions for the bigger group over the field with $p^{2}$ elements. Using the calculations and notation in [Ye1, one can show the following for the finite groups $S p_{4}\left(\mathbb{F}_{q}\right)$.

Example. Let $p \geq 5$ and $\lambda, \mu \in X_{r}(T)$. Then $\operatorname{dim}_{k} \operatorname{Ext}_{S p_{4}\left(\mathbb{F}_{q}\right)}^{1}(L(\lambda), L(\mu)) \leq 1$, unless $r=2$ and $\lambda_{1}=\mu_{0}=(s,(p-1) / 2)$ and $\lambda_{0}=\mu_{1}=(s+1,(p-3) / 2)$ or $\lambda_{1}=\mu_{0}=(s+1,(p-3) / 2)$ and $\lambda_{0}=\mu_{1}=(s,(p-1) / 2)$, for $1 \leq s \leq p-2$. In that case the dimension is 2 . 
3.4. In the previous sections, it was shown that for $r \geq 2$, there exists an algorithm for obtaining the extensions of two given simple modules for the finite group from the extensions of simple modules for the ambient algebraic group. We now present a slightly different point of view. For a fixed simple module $L(\lambda)$ with $\lambda \in X_{r}(T)$, there is a one-to-one correspondence between the set of simple modules for the finite group $G\left(\mathbb{F}_{q}\right)$ which have $p^{r}$-restricted highest weight and extend $L(\lambda)$ nontrivially and a set of $p^{r+1}$-restricted highest weights whose corresponding simple modules admit certain nontrivial extensions over $G$. This is presented in the following theorem, which greatly improves [Pil, Lem. 2.2] for $r \geq 2$.

Theorem. Let $G$ be a simple simply connected algebraic group with $p \geq 3(h-1)$ and $r \geq 2$. Furthermore, assume that if the underlying root system is of type $A_{1}$ or $C_{n}$, then $r \geq 3$. For $\lambda \in X_{r}(T)$ define the sets

$$
\begin{aligned}
A(\lambda)=\left\{\mu+p^{r} \mu_{r} \mid\right. & \mu \in X_{r}(T), \mu_{r} \in X_{1}(T), \mu \neq \lambda, \\
& \left.\operatorname{Ext}_{G}^{1}\left(L\left(\lambda+p^{r} \lambda_{0}\right), L\left(\mu+p^{r} \mu_{r}\right)\right) \neq 0\right\}
\end{aligned}
$$

and

$$
B(\lambda)=\left\{\sigma \in X_{r}(T) \mid \operatorname{Ext}_{G\left(\mathbb{F}_{q}\right)}^{1}(L(\lambda), L(\sigma)) \neq 0\right\},
$$

and a map $\phi: A(\lambda) \rightarrow X(T)$ with $\phi\left(\mu+p^{r} \mu_{r}\right)=\mu+\mu_{r}-\lambda_{0}$. Then $\phi$ maps $A(\lambda)$ bijectively onto $B(\lambda)$, and for any $\mu+p^{r} \mu_{r} \in A(\lambda)$ one obtains

$$
\operatorname{Ext}_{G}^{1}\left(L\left(\lambda+p^{r} \lambda_{0}\right), L\left(\mu+p^{r} \mu_{r}\right)\right) \cong \operatorname{Ext}_{G\left(\mathbb{F}_{q}\right)}^{1}\left(L(\lambda), L\left(\phi\left(\mu+p^{r} \mu_{r}\right)\right)\right) .
$$

Proof. We will first show that $\phi(A(\lambda)) \subset B(\lambda)$. Let $\mu+p^{r} \mu_{r} \in A(\lambda)$. So $\operatorname{Ext}_{G}^{1}\left(L\left(\lambda+p^{r} \lambda_{0}\right), L\left(\mu+p^{r} \mu\right)\right) \neq 0$ and we must show that $\operatorname{Ext}_{G}^{1}(L(\lambda), L(\mu+$ $\left.\left.\mu_{r}-\lambda_{0}\right)\right) \neq 0$.

Case 1.1. $\mu_{0}=\lambda_{0}$.

We define $\lambda_{r}=\lambda_{0}, \widetilde{\lambda}=\sum_{i=1}^{r} p^{i-1} \lambda_{i}$, and $\widetilde{\mu}=\sum_{i=1}^{r} p^{i-1} \mu_{i}$. Since $\lambda+p^{r} \lambda_{0}=$ $\lambda_{0}+p \widetilde{\lambda}$ and $\mu+p^{r} \mu_{r}=\lambda_{0}+p \widetilde{\mu}$, by Lemma 2.7(A), $\operatorname{Ext}_{G}^{1}\left(L\left(\lambda+p^{r} \lambda_{0}\right), L(\mu+\right.$ $\left.\left.p^{r} \mu_{r}\right)\right) \cong \operatorname{Ext}_{G}^{1}(L(\widetilde{\lambda}), L(\widetilde{\mu}))$. Further, the restriction map $\operatorname{Ext}_{G}^{1}(L(\widetilde{\lambda}), L(\widetilde{\mu}))$ to $\operatorname{Ext}_{G\left(\mathbb{F}_{q}\right)}^{1}(L(\widetilde{\lambda}), L(\widetilde{\mu}))$ is an injection. Finally, as $G\left(\mathbb{F}_{q}\right)$-modules, $L(\widetilde{\lambda})^{(1)} \cong L(\lambda)$ and $L(\widetilde{\mu})^{(1)} \cong L\left(\mu+\mu_{r}-\mu_{0}\right)=L\left(\mu+\mu_{r}-\lambda_{0}\right)$. Hence,

$$
\begin{aligned}
\operatorname{Ext}_{G}^{1}\left(L\left(\lambda+p^{r} \lambda_{0}\right), L\left(\mu+p^{r} \mu_{r}\right)\right) & \hookrightarrow \operatorname{Ext}_{G\left(\mathbb{F}_{q}\right)}^{1}(L(\widetilde{\lambda}), L(\widetilde{\mu})) \\
& \cong \operatorname{Ext}_{G\left(\mathbb{F}_{q}\right)}^{1}\left(L(\lambda), L\left(\mu+\mu_{r}-\lambda_{0}\right)\right),
\end{aligned}
$$

and $\phi\left(\mu+p^{r} \mu_{r}\right) \in B(\lambda)$.

Case 1.2. $\mu_{0} \neq \lambda_{0}$.

Since $\operatorname{Ext}_{G}^{1}\left(L\left(\lambda+p^{r} \lambda_{0}\right), L\left(\mu+p^{r} \mu_{r}\right)\right) \neq 0$, it follows from Corollary 2.4(a) that $\mu_{r}=\lambda_{0}$ and so $\phi\left(\mu+p^{r} \mu_{r}\right)=\mu$. Furthermore, by Lemma 2.7(A), $\operatorname{Ext}_{G}^{1}\left(L\left(\lambda+p^{r} \lambda_{0}\right), L\left(\mu+p^{r} \mu_{r}\right)\right) \cong \operatorname{Ext}_{G}^{1}(L(\lambda), L(\mu))$. Again the restriction map from $\operatorname{Ext}_{G}^{1}(L(\lambda), L(\mu))$ to $\operatorname{Ext}_{G\left(\mathbb{F}_{q}\right)}^{1}(L(\lambda), L(\mu))$ is injective. Now $\phi\left(\mu+p^{r} \mu_{r}\right) \in B(\lambda)$ because

$$
\begin{aligned}
\operatorname{Ext}_{G}^{1}\left(L\left(\lambda+p^{r} \lambda_{0}\right), L\left(\mu+p^{r} \mu_{r}\right)\right) & \hookrightarrow \operatorname{Ext}_{G\left(\mathbb{F}_{q}\right)}^{1}(L(\lambda), L(\mu)) \\
& =\operatorname{Ext}_{G\left(\mathbb{F}_{q}\right)}^{1}\left(L(\lambda), L\left(\mu+\mu_{r}-\lambda_{0}\right)\right) .
\end{aligned}
$$


Next we will show that $\phi$ maps $A(\lambda)$ onto $B(\lambda)$. Specifically, we will show that for each $\sigma \in B(\lambda)$ there exists a $\mu+p^{r} \mu_{r} \in A(\lambda)$ such that $\phi\left(\mu+p^{r} \mu_{r}\right)=\sigma$ and moreover that

$$
\operatorname{Ext}_{G}^{1}\left(L\left(\lambda+p^{r} \lambda_{0}\right), L\left(\mu+p^{r} \mu_{r}\right)\right) \cong \operatorname{Ext}_{G\left(\mathbb{F}_{q}\right)}^{1}(L(\lambda), L(\sigma)) .
$$

Case 2.1. $\lambda_{r-1}=\sigma_{r-1}$.

Since $\operatorname{Ext}_{G_{1}}^{1}\left(L\left(\lambda_{r-1}\right), L\left(\sigma_{r-1}\right)\right)=0$, Theorem 2.1(b) implies that

$$
\operatorname{Ext}_{G\left(\mathbb{F}_{q}\right)}^{1}(L(\lambda), L(\sigma)) \cong \operatorname{Ext}_{G}^{1}(L(\lambda), L(\sigma))
$$

Since $\lambda_{r-1}=\sigma_{r-1}$, we may apply Lemma 2.7(A) to conclude that $\operatorname{Ext}_{G}^{1}(L(\lambda), L(\sigma))$ $\cong \operatorname{Ext}_{G}^{1}\left(L\left(\lambda+p^{r} \lambda_{0}\right), L\left(\sigma+p^{r} \lambda_{0}\right)\right)$. Set $\mu_{r}=\lambda_{0}$ and $\mu=\sigma$. Then $\mu+p^{r} \mu_{r}=\sigma+p^{r} \lambda_{0}$. So $\phi\left(\mu+p^{r} \mu_{r}\right)=\sigma$ and $\operatorname{Ext}_{G}^{1}\left(L\left(\lambda+p^{r} \lambda_{0}\right), L\left(\mu+p^{r} \mu_{r}\right)\right) \cong \operatorname{Ext}_{G\left(\mathbb{F}_{q}\right)}^{1}(L(\lambda), L(\sigma))$.

Case 2.2. $\lambda_{r-1} \neq \sigma_{r-1}$ and $\lambda_{r-2}=\sigma_{r-2}$.

We set $\lambda_{r}=\lambda_{0}, \sigma_{r}=\sigma_{0}, \tilde{\lambda}=\sum_{i=1}^{r} p^{i-1} \lambda_{i}$, and $\widetilde{\sigma}=\sum_{i=1}^{r} p^{i-1} \sigma_{i}$. Then $L(\widetilde{\lambda})^{(1)} \cong L(\lambda)$ and $L(\widetilde{\sigma})^{(1)} \cong L(\sigma)$ as $G\left(\mathbb{F}_{q}\right)$-modules; so $\operatorname{Ext}_{G\left(\mathbb{F}_{q}\right)}^{1}(L(\lambda), L(\sigma)) \cong$ $\operatorname{Ext}_{G\left(\mathbb{F}_{q}\right)}^{1}(L(\widetilde{\lambda}), L(\widetilde{\sigma}))$. Furthermore, $\operatorname{Ext}_{G\left(\mathbb{F}_{q}\right)}^{1}(L(\widetilde{\lambda}), L(\widetilde{\sigma})) \cong \operatorname{Ext}_{G}^{1}(L(\widetilde{\lambda}), L(\widetilde{\sigma}))$. For $r \geq 3$, this follows from Theorem 2.4(b) since $R=0$ as $\widetilde{\lambda}_{r-2} \neq \widetilde{\sigma}_{r-2}$ implies $\ddot{\widetilde{\lambda}} \neq \ddot{\widetilde{\sigma}}$. For $r=2$, the isomorphism follows from Theorem $2.1(\mathrm{~b})$ since $\widetilde{\lambda}_{r-1}=\widetilde{\sigma}_{r-1}$ and $\operatorname{Ext}_{G_{1}}\left(L\left(\widetilde{\lambda}_{r-1}\right), L\left(\widetilde{\sigma}_{r-1}\right)\right)=0$.

On the other hand, by Lemma $2.7(\mathrm{~A})$, we have

$\operatorname{Ext}_{G}^{1}(L(\widetilde{\lambda}), L(\widetilde{\sigma})) \cong \operatorname{Ext}_{G}^{1}\left(L\left(\lambda_{0}+p \widetilde{\lambda}\right), L\left(\lambda_{0}+p \widetilde{\sigma}\right)\right)=\operatorname{Ext}_{G}^{1}\left(L\left(\lambda+p^{r} \lambda_{0}\right), L\left(\lambda_{0}+p \widetilde{\sigma}\right)\right)$. Set $\mu_{r}=\sigma_{0}$ and $\mu=\sigma-\sigma_{0}+\lambda_{0}$. Then $\mu+p^{r} \mu_{r}=\sigma-\sigma_{0}+\lambda_{0}+p^{r} \sigma_{0}=\lambda_{0}+p \widetilde{\sigma}$, $\phi\left(\mu+p^{r} \mu_{r}\right)=\sigma-\sigma_{0}+\lambda_{0}+\sigma_{0}-\lambda_{0}=\sigma$, and $\operatorname{Ext}_{G}^{1}\left(L\left(\lambda+p^{r} \lambda_{0}\right), L\left(\mu+p^{r} \mu_{r}\right)\right) \cong$ $\operatorname{Ext}_{G\left(\mathbb{F}_{q}\right)}^{1}(L(\lambda), L(\sigma))$.

Case 2.3. $\lambda_{r-1} \neq \sigma_{r-1}$ and $\lambda_{r-2} \neq \sigma_{r-2}$.

If $r \geq 3$, apply Theorem 2.4(b) to $\lambda$ and $\sigma$. By assumption, $\ddot{\lambda} \neq \ddot{\sigma}$. So $R=$ 0 and $\operatorname{Ext}_{G\left(\mathbb{F}_{q}\right)}^{1}(L(\lambda), L(\sigma)) \cong \operatorname{Ext}_{G}^{1}(L(\lambda), L(\sigma))$. Moreover, by Lemma 2.7(A), $\operatorname{Ext}_{G}^{1}(L(\lambda), L(\sigma)) \cong \operatorname{Ext}_{G}^{1}\left(L\left(\lambda+p^{r} \lambda_{0}\right), L\left(\sigma+p^{r} \lambda_{0}\right)\right)$. As in Case 2.1, we choose $\mu_{r}=\lambda_{0}$ and $\mu=\sigma$ to obtain the isomorphism.

If $r=2$, then by Theorem 3.1(c),

$$
\operatorname{Ext}_{G\left(\mathbb{F}_{q}\right)}^{1}(L(\lambda), L(\sigma)) \cong \operatorname{Ext}_{G}^{1}(L(\lambda), L(\sigma)) \oplus \operatorname{Ext}_{G}^{1}(L(\hat{\lambda}), L(\hat{\sigma})) .
$$

However, it follows from the proof of Theorem 3.3 that, provided $\Phi$ is not of type $A_{1}$ or $C_{n}$, only one of these terms is nonzero. If $\operatorname{Ext}_{G\left(\mathbb{F}_{q}\right)}^{1}(L(\lambda), L(\sigma)) \cong$ $\operatorname{Ext}_{G}^{1}(L(\lambda), L(\sigma))$, we may proceed as we did for $r \geq 3$ and set $\mu_{r}=\lambda_{0}$ and $\mu=\sigma$. If $\operatorname{Ext}_{G\left(\mathbb{F}_{q}\right)}^{1}(L(\lambda), L(\sigma)) \cong \operatorname{Ext}_{G}^{1}(L(\hat{\lambda}), L(\hat{\sigma}))$, we may proceed as in Case 2.2 and set $\mu_{r}=\sigma_{0}$ and $\mu=\sigma-\sigma_{0}+\lambda_{0}$.

Finally, we need to show that $\phi$ is one-to-one. Assume that $\mu+p^{r} \mu_{r} \in A(\lambda)$ and $\delta+p^{r} \delta_{r} \in A(\lambda)$ such that $\mu+p^{r} \mu_{r} \neq \delta+p^{r} \delta_{r}$ while $\mu+\mu_{r}-\lambda_{0}=\delta+\delta_{r}-\lambda_{0}$. It follows immediately that $\mu_{0} \neq \delta_{0}$ and $\mu_{r} \neq \delta_{r}$. Because $\mu_{0} \neq \delta_{0}$ we may assume without loss of generality that $\mu_{0} \neq \lambda_{0}$. Applying Corollary 2.4(a) to $\operatorname{Ext}_{G}^{1}\left(L\left(\lambda+p^{r} \lambda_{0}\right), L\left(\mu+p^{r} \mu_{r}\right)\right)$, it follows that $\mu_{i}=\lambda_{i}$ for $2 \leq i \leq r-1$ and $\mu_{r}=\lambda_{0}$. Then, since $\delta_{r} \neq \mu_{r}=\lambda_{0}$, it similarly follows from Corollary 2.4(a) that $\delta_{i}=\lambda_{i}$ for $0 \leq i \leq r-2$. In particular, $\delta_{0}=\lambda_{0}=\mu_{r}$. Then from the equation 
$\mu+\mu_{r}=\delta+\delta_{r}$, we conclude that $\mu=\delta-\delta_{0}+\delta_{r}$. Hence, $\mu_{0}=\delta_{r}$ and $\mu_{i}=\delta_{i}$ for $1 \leq i \leq r-1$. If $r \geq 3$, then $r-1 \geq 2$ and so $\delta_{r-1}=\mu_{r-1}=\lambda_{r-1}$ and therefore $\delta=\lambda$. But that violates the condition $\delta \neq \lambda$ for elements $\delta+p^{r} \delta_{r}$ in $A(\lambda)$. If $r=2$, $\delta_{1}=\mu_{1}$ need not equal $\lambda_{1}$. If it does, we arrive at the same contradiction. In any case, as in Theorem 3.1, let $\hat{\lambda}=\lambda_{1}+p \lambda_{0}$ and $\hat{\mu}=\mu_{1}+p \mu_{0}$. Since $\mu_{2}=\lambda_{0}$ and $\lambda_{0} \neq \mu_{0}$, it follows from Lemma 2.7(A) that

$$
\begin{aligned}
\operatorname{Ext}_{G}^{1}(L(\lambda), L(\mu)) & \cong \operatorname{Ext}_{G}^{1}\left(L\left(\lambda+p^{2} \lambda_{0}\right), L\left(\mu+p^{2} \lambda_{0}\right)\right) \\
& =\operatorname{Ext}_{G}^{1}\left(L\left(\lambda+p^{2} \lambda_{0}\right), L\left(\mu+p^{2} \mu_{2}\right)\right) \neq 0 .
\end{aligned}
$$

On the other hand, since $\delta_{0}=\lambda_{0}, \delta_{1}=\mu_{1}$, and $\delta_{2}=\mu_{0}$, we have $\delta+p^{2} \delta_{2}=\lambda_{0}+p \hat{\mu}$. Hence, we also have

$$
\begin{aligned}
\operatorname{Ext}_{G}^{1}(L(\hat{\lambda}), L(\hat{\mu})) & \cong \operatorname{Ext}_{G}^{1}\left(L\left(\lambda_{0}+p \hat{\lambda}\right), L\left(\lambda_{0}+p \hat{\mu}\right)\right) \\
& =\operatorname{Ext}_{G}^{1}\left(L\left(\lambda+p^{2} \lambda_{0}\right), L\left(\delta+p^{2} \delta_{2}\right)\right) \neq 0 .
\end{aligned}
$$

But, as shown in the proof of Theorem 3.3, $\operatorname{Ext}_{G}^{1}(L(\lambda), L(\mu))$ and $\operatorname{Ext}_{G}^{1}(L(\hat{\lambda}), L(\hat{\mu}))$ cannot both vanish unless $\Phi$ is of type $A_{1}$ or $C_{n}$. Hence, $\phi$ is one-to-one.

The example in Section 3.3 as well as AJL Cor. 4.5 (b)] shows that the theorem above fails for certain weights $\lambda$ and groups of type $A_{1}$ or $C_{2}$ and $r=2$. The map $\phi$ is not one-to-one in these cases.

\section{Cohomology For finite AND ALgebraic Groups}

4.1. In this section, we turn to the special case of finding relationships between cohomology groups over $G\left(\mathbb{F}_{q}\right), G$, and $G_{r}$. Here $T_{\gamma}^{\sigma}$ denotes Jantzen's translation functor [Jan1 II.7]. We begin by providing a generalization of [BNP1, Cor. 7.4].

Proposition. Let $G$ be a simple simply connected algebraic group with $p \geq$ $(2 s+1)(h-1)$ for a positive integer $s$, and let $V \in \mathcal{C}_{s, r}$ have only $p^{r}$-restricted composition factors in its socle (as a G-module). Then for $0 \leq i \leq s$,

$$
\mathrm{H}^{i}\left(G\left(\mathbb{F}_{q}\right), V\right) \cong \bigoplus_{\nu \in \Gamma_{2 s(h-1)}} \mathrm{H}^{i}\left(G, T_{\nu}^{0}\left(V \otimes L(\nu)^{(r)}\right)\right)
$$

Proof. Since $p \geq(2 s+1)(h-1)$ and $\nu \in \Gamma_{2 s(h-1)}$, it follows that $\nu$ is in the interior of the lowest alcove. Consequently, from Jantzen's translation principle, 
$L(\nu) \cong T_{0}^{\nu}(k)$. One can now argue as follows:

$$
\begin{aligned}
\mathrm{H}^{i}\left(G\left(\mathbb{F}_{q}\right), V\right) & \cong \operatorname{Ext}_{G\left(\mathbb{F}_{q}\right)}^{i}(k, V) \\
& \cong \operatorname{Ext}_{G\left(\mathbb{F}_{q}\right)}^{i}\left(V^{*}, k\right) \\
& \cong \operatorname{Ext}_{G}^{i}\left(V^{*}, \mathcal{G}_{s}(k)\right) \quad([\text { BNP1, Thm. 5.2]) } \\
& \cong \bigoplus_{\nu \in \Gamma_{2 s(h-1)}} \operatorname{Ext}_{G}^{i}\left(V^{*}, L\left(\left(p^{r}-w_{0}\right) \nu\right)\right) \quad([\text { BNP1, Thm. 7.4] }) \\
& \cong \bigoplus_{\nu \in \Gamma_{2 s(h-1)}} \operatorname{Ext}_{G}^{i}\left(L(\nu), V \otimes L(\nu)^{(r)}\right) \quad([\text { [Jan1, I.4.4] }) \\
& \cong \bigoplus_{\nu \in \Gamma_{2 s(h-1)}} \operatorname{Ext}_{G}^{i}\left(T_{0}^{\nu}(k), V \otimes L(\nu)^{(r)}\right) \\
& \cong \bigoplus_{\nu \in \Gamma_{2 s(h-1)}} \operatorname{Ext}_{G}^{i}\left(k, T_{\nu}^{0}\left(V \otimes L(\nu)^{(r)}\right)\right) \quad([\underline{\text { Jan1 }}, \text { II.7.6] }) \\
& \cong \bigoplus_{\nu \in \Gamma_{2 s(h-1)}} \mathrm{H}^{i}\left(G, T_{\nu}^{0}\left(V \otimes L(\nu)^{(r)}\right)\right) .
\end{aligned}
$$

4.2. A weight $\lambda$ is $G_{1}$-linked to the zero weight if $\lambda=w \cdot 0+p \gamma$ for some $w \in W$ and $\gamma \in X(T)$. The result below shows that cohomology for the algebraic group and the finite group coincide if the $G$-module $V$ has all its $G_{1}$-composition factors in the principal block for $G_{1}$.

Corollary (A). Let $G$ be a simple simply connected algebraic group with $p>$ $(2 s+1)(h-1)$ for a positive integer $s$. Suppose that $V \in \mathcal{C}_{s, r}$ has only $p^{r}$ restricted composition factors in its socle (as a $G$-module). If all the highest weights of the simple $G_{1}$-composition factors of $V$ are $G_{1}$-linked to the zero weight, then for $0 \leq i \leq s$,

$$
\mathrm{H}^{i}\left(G\left(\mathbb{F}_{q}\right), V\right) \cong \mathrm{H}^{i}(G, V) .
$$

Proof. According to Proposition 4.1, we have

$$
\mathrm{H}^{i}\left(G\left(\mathbb{F}_{q}\right), V\right) \cong \bigoplus_{\nu \in \Gamma_{2 s(h-1)}} \mathrm{H}^{i}\left(G, T_{\nu}^{0}\left(V \otimes L(\nu)^{(r)}\right)\right) .
$$

Suppose that there exists a nonzero $\nu \in \Gamma_{2 s(h-1)}$ and a composition factor $L(\lambda)$ of $V$ with $T_{\nu}^{0}\left(L(\lambda) \otimes L(\nu)^{(r)}\right) \neq 0$. Then $\lambda$ is $G_{1}$-linked to $\nu$. On the other hand, $\lambda$ is also $G_{1}$-linked to zero. Following [Jan2, Lem. 1] there exists a $w \in W$ and a corresponding unique weight $\rho_{w} \in X(T)_{+}$such that $0=w \cdot \nu+p \rho_{w}$. One obtains $w(\nu+\rho)-\rho=-p \rho_{w}$. Taking the absolute value of the inner product of the left-hand side with a simple root $\alpha$ yields

$$
\left|\left\langle w(\nu+\rho), \alpha^{\vee}\right\rangle-1\right| \leq 2 s(h-1)-1+(h-1)+1=(2 s+1)(h-1),
$$

which is strictly less than $p$. Therefore, $\rho_{w}=0, w=1$ and $\nu=0$. We obtain a contradiction and the assertion follows.

We can now apply Proposition 4.1 and Corollary 4.2(A) to simple modules and induced $G$-modules. Recall that a weight $\lambda$ is called $p$-regular if $\left\langle\lambda+\rho, \alpha^{\vee}\right\rangle$ is not divisible by $p$ for any root $\alpha$. Otherwise we say $\lambda$ is $p$-singular. 
Corollary (B). Let $G$ be a simple simply connected algebraic group with $p>$ $(2 s+1)(h-1)$ for a positive integer $s$ and $\lambda \in X_{r}(T)$. For $0 \leq i \leq s$, the following hold:

(i) If $\lambda$ is p-singular, then $\mathrm{H}^{i}\left(G\left(\mathbb{F}_{q}\right), H^{0}(\lambda)\right) \cong \mathrm{H}^{i}\left(G\left(\mathbb{F}_{q}\right), L(\lambda)\right)=0$.

(ii) If $\lambda$ is $G_{1}$-linked to the zero weight, then $\mathrm{H}^{i}\left(G\left(\mathbb{F}_{q}\right), L(\lambda)\right) \cong \mathrm{H}^{i}(G, L(\lambda))$.

(iii) If $\lambda$ is $G_{1}$-linked to the zero weight, then $\mathrm{H}^{i}\left(G\left(\mathbb{F}_{q}\right), H^{0}(\lambda)\right)=0$.

Proof. (i) The size of $p$ and the definition of the set $\Gamma_{2 s(h-1)}$ force all the weights $\nu$ that appear in the sum in equation (4.1.1) to be $p$-regular. Therefore, for any $\nu$ and for any $p$-singular $\lambda, T_{\nu}^{0}\left(H^{0}(\lambda) \otimes L(\nu)^{(r)}\right) \cong T_{\nu}^{0}\left(L^{0}(\lambda) \otimes L(\nu)^{(r)}\right)=0$.

(ii) This follows immediately from Corollary $4.2(\mathrm{~A})$.

(iii) Again, it is immediate from Corollary $4.2(\mathrm{~A})$ that $\mathrm{H}^{i}\left(G\left(\mathbb{F}_{q}\right), H^{0}(\lambda)\right) \cong$ $\mathrm{H}^{i}\left(G, H^{0}(\lambda)\right)$. Now apply [Jan1, II.4.13].

4.3. The following proposition is essential for our computations and will be used throughout the remainder of this section. This result is a consequence of the computation of $G_{1}$-cohomology with coefficients in an induced module (see [A.J], [KLT]). Andersen And2] extended these results to the first cohomology of higher Frobenius kernels. We note that it is possible to extend some of the work of Jantzen [Jan5] for cohomology groups of $G_{1}$ to $G_{r}$ and replace the condition $p>h$ with $p>3$.

Proposition. Let $G$ be a simple algebraic group with $p>h$. Let $\nu, \zeta \in X(T)_{+}$.

(a) Then

$$
\begin{aligned}
& \operatorname{Hom}_{G}\left(L(\nu)^{(r)}, \operatorname{Ext}_{G_{r}}^{1}\left(k, H^{0}(\zeta)\right)\right) \\
& \quad= \begin{cases}k & \text { if } \zeta=p^{r} \nu-p^{i} \alpha \text { for some } \alpha \in \Delta \text { and } i<r, \\
0 & \text { else. }\end{cases}
\end{aligned}
$$

(b) Moreover, if $\zeta \in X_{r}(T)$ and either $\zeta \neq 0$ or $\nu \in \bar{C}_{\mathbb{Z}}$, then

$\operatorname{Ext}_{G}^{1}\left(L(\nu)^{(r)}, H^{0}(\zeta)\right)= \begin{cases}k & \begin{array}{l}\text { if } \zeta=p^{r} \omega_{\alpha}-p^{i} \alpha \text { and } \nu=\omega_{\alpha} \text { where } \alpha \in \Delta, \omega_{\alpha} \text { is } \\ \text { the corresponding fundamental weight, and } i<r, \\ \text { else. }\end{array}\end{cases}$

Proof. (a) According to And2, p. 392],

$$
\operatorname{Ext}_{G_{r}}^{1}\left(k, H^{0}(\zeta)\right)= \begin{cases}H^{0}(\delta)^{(r)} & \text { if } \zeta=p^{r} \delta-p^{i} \alpha \text { for some } \alpha \in \Delta \text { and } i<r, \\ 0 & \text { else. }\end{cases}
$$

Hence, if $\operatorname{Ext}_{G_{r}}^{1}\left(k, H^{0}(\zeta)\right) \neq 0$, then

$$
\operatorname{Hom}_{G}\left(L(\nu)^{(r)}, \operatorname{Ext}_{G_{r}}^{1}\left(k, H^{0}(\zeta)\right)\right) \cong \operatorname{Hom}_{G}\left(L(\nu)^{(r)}, H^{0}(\delta)^{(r)}\right) .
$$

But, this Hom-group is nonzero and equal to $k$ if and only if $\delta=\nu$.

(b) We now apply the Lyndon-Hochschild-Serre spectral sequence

$$
\operatorname{Ext}_{G / G_{r}}^{i}\left(L(\nu)^{r}, \operatorname{Ext}_{G_{r}}^{j}\left(k, H^{0}(\zeta)\right)\right) \Rightarrow \operatorname{Ext}_{G}^{i+j}\left(L(\nu)^{(r)}, H^{0}(\zeta)\right)
$$

with the corresponding five term exact sequence

$$
0 \rightarrow E_{2}^{1,0} \rightarrow E^{1} \rightarrow E_{2}^{0,1} \rightarrow E_{2}^{2,0} \rightarrow E^{2} .
$$

If $\zeta \in X_{r}(T)$, then

$$
\operatorname{Hom}_{G_{r}}\left(k, H^{0}(\zeta)\right)= \begin{cases}k & \text { if } \zeta=0 \\ 0 & \text { else. }\end{cases}
$$


Since $\zeta \neq 0$ or $\nu \in \bar{C}_{\mathbb{Z}}$, it follows that for $i>0$,

$$
E^{i, 0}=\operatorname{Ext}_{G / G_{r}}^{i}\left(L(\nu)^{(r)}, \operatorname{Hom}_{G_{r}}\left(k, H^{0}(\zeta)\right)\right)=0 .
$$

Therefore, we obtain the isomorphism

$$
\operatorname{Ext}_{G}^{1}\left(L(\nu)^{(r)}, H^{0}(\zeta)\right) \cong \operatorname{Hom}_{G / G_{r}}\left(L(\nu)^{(r)}, \operatorname{Ext}_{G_{r}}^{1}\left(k, H^{0}(\zeta)\right)\right) .
$$

By part (a) of this proposition, this is nonzero if and only if $\zeta=p^{r} \nu-p^{i} \alpha$. Since $\zeta$ is $p^{r}$-restricted, for any simple $\operatorname{root} \beta$ with $\left\langle\alpha, \beta^{\vee}\right\rangle \leq 0$, one concludes that $\left\langle\nu, \beta^{\vee}\right\rangle=0$, while clearly $\left\langle\nu, \alpha^{\vee}\right\rangle=1$. Hence, $\nu=\omega_{\alpha}$.

4.4. In [FP, Thm. 3.3] Friedlander and Parshall compute the dimensions of the $G\left(\mathbb{F}_{q}\right)$-cohomology groups $\mathrm{H}^{i}\left(G\left(\mathbb{F}_{q}\right), H^{0}(\lambda)\right)$ for induced $G$-modules, provided the prime is sufficiently large with respect to $G, \lambda$, and $i$. In particular, they prove that the odd cohomology groups vanish in these cases. The following gives a complete description of the first $G\left(\mathbb{F}_{q}\right)$-cohomology of induced $G$-modules with $p^{r}$-restricted highest weights for $p \geq 3(h-1)$. One observes nonvanishing for certain weights outside of the region considered in [FP].

Theorem. Let $G$ be a simple simply connected algebraic group with $p \geq 3(h-1)$. Let $\lambda \in X_{r}(T)$. Then

$$
\mathrm{H}^{1}\left(G\left(\mathbb{F}_{q}\right), H^{0}(\lambda)\right)= \begin{cases}k \quad \begin{array}{l}
\text { if } \lambda=p^{r} \omega_{\alpha}-p^{i} \alpha-w_{0} \omega_{\alpha} \text { where } \alpha \in \Delta, \omega_{\alpha} \text { the } \\
\text { corresponding fundamental weight, and } 1 \leq i<r,
\end{array} \\
k \quad \begin{array}{l}
\text { if } \lambda=p^{r} \omega_{\alpha}+s_{\alpha} \cdot\left(-w_{0} \omega_{\alpha}\right) \text { where } \alpha \in \Delta, \omega_{\alpha} \text { the } \\
\text { corresponding fundamental weight, }
\end{array} \\
0 \quad \text { else. }\end{cases}
$$

Proof. According to Proposition 4.1 with $V=H^{0}(\lambda)$,

$$
\begin{aligned}
\mathrm{H}^{1}\left(G\left(\mathbb{F}_{q}\right), H^{0}(\lambda)\right) & \cong \bigoplus_{\nu \in \Gamma_{2(h-1)}} \mathrm{H}^{1}\left(G, T_{\nu}^{0}\left(H^{0}(\lambda) \otimes L(\nu)^{(r)}\right)\right) \\
& \cong \bigoplus_{\nu \in \Gamma_{2(h-1)}} \operatorname{Ext}_{G}^{1}\left(L\left(p^{r} \nu\right), T_{\left(p^{r}-w_{0}\right) \nu}^{p^{r} \nu}\left(H^{0}(\lambda)\right)\right) .
\end{aligned}
$$

We fix some $\nu$ with $\operatorname{Ext}_{G}^{1}\left(L\left(p^{r} \nu\right), T_{\left(p^{r}-w_{0}\right) \nu}^{p^{r} \nu}\left(H^{0}(\lambda)\right)\right) \neq 0$ and define the $p^{r}$-restricted weight $\mu$ via

$$
H^{0}(\mu)=T_{\left(p^{r}-w_{0}\right) \nu}^{p^{r} \nu}\left(H^{0}(\lambda)\right)
$$

Notice that all $\nu \in \Gamma_{2(h-1)}$ are also contained in $\bar{C}_{\mathbb{Z}}$. According to Proposition 4.3(b), $\operatorname{Ext}_{G}^{1}\left(L\left(p^{r} \nu\right), H^{0}(\mu)\right)=0$, unless $\nu=\omega_{\alpha}$ and $\mu=p^{r} \omega_{\alpha}-p^{i} \alpha$ where $\alpha \in \Delta$, $\omega_{\alpha}$ is the corresponding fundamental weight, and $i<r$. In that case it is equal to $k$. We translate back and find that

$$
\lambda= \begin{cases}p^{r} \omega_{\alpha}-p^{i} \alpha-w_{0} \omega_{\alpha} & \text { for } 0<i, \\ p^{r} \omega_{\alpha}+s_{\alpha} \cdot\left(-w_{0} \omega_{\alpha}\right) & \text { for } i=0 .\end{cases}
$$

It is left to show that two distinct weights $\omega_{\alpha}, \omega_{\beta}$ cannot contribute in the summation for the same $\lambda$. Assume the contrary. Then the weights $\omega_{\alpha}$ and $\omega_{\beta}$ are $G_{1}$-linked. That is, $\omega_{\alpha}=w \cdot \omega_{\beta}+p \gamma$ for some $w \in W$ and some $\gamma \in X(T)$. Thus $\left\langle\omega_{\alpha}, \beta^{\vee}\right\rangle-\left\langle w \cdot \omega_{\beta}, \beta^{\vee}\right\rangle$ has to be divisible by $p$. Now $\left|\left\langle\omega_{\alpha}, \beta^{\vee}\right\rangle-\left\langle w \cdot \omega_{\beta}, \beta^{\vee}\right\rangle\right| \leq$ $2(h-1)+1$. That forces $\left|\left\langle\omega_{\alpha}, \beta^{\vee}\right\rangle-\left\langle w \cdot \omega_{\beta}, \beta^{\vee}\right\rangle\right|=0$, unless $p=3$ and $\Phi$ is of 
type $A_{1}$. But in that case the only choice is $\omega_{\alpha}=\omega_{\beta}=1$. Therefore, $\gamma=0$ and $\omega_{\alpha}=w \cdot \omega_{\beta}$. It follows that $\alpha=\beta$.

4.5. The following proposition provides a computation of the extension between certain simple $G$-modules. This computation will be used in the upcoming sections.

Proposition. Let $G$ be a simple algebraic group with $p>h$. For any simple root $\alpha$ with corresponding fundamental weight $\omega_{\alpha}$, we obtain

$$
\operatorname{Ext}_{G}^{1}\left(L\left(\omega_{\alpha}\right)^{(1)}, L\left(p \omega_{\alpha}-\alpha\right)\right) \cong k
$$

Proof. Since $p>h$ it follows that $p \omega_{\alpha}-\alpha$ is not the zero weight. From Proposition 4.3(b), $\operatorname{Ext}_{G}^{1}\left(L\left(\omega_{\alpha}\right)^{(1)}, H^{0}\left(p \omega_{\alpha}-\alpha\right)\right) \cong k$ and so we proceed to show that

$$
\operatorname{Ext}_{G}^{1}\left(L\left(p \omega_{\alpha}\right), L\left(p \omega_{\alpha}-\alpha\right)\right) \cong \operatorname{Ext}_{G}^{1}\left(L\left(p \omega_{\alpha}\right), H^{0}\left(p \omega_{\alpha}-\alpha\right)\right)
$$

Define the $G$-module $Q$ via the exact sequence

$$
0 \rightarrow L\left(p \omega_{\alpha}-\alpha\right) \rightarrow H^{0}\left(p \omega_{\alpha}-\alpha\right) \rightarrow Q \rightarrow 0
$$

in order to obtain the long exact sequence

$$
\begin{aligned}
\cdots & \rightarrow \operatorname{Hom}_{G}\left(L\left(p \omega_{\alpha}\right), Q\right) \rightarrow \operatorname{Ext}_{G}^{1}\left(L\left(p \omega_{\alpha}\right), L\left(p \omega_{\alpha}-\alpha\right)\right) \\
& \rightarrow \operatorname{Ext}_{G}^{1}\left(L\left(p \omega_{\alpha}\right), H^{0}\left(p \omega_{\alpha}-\alpha\right)\right) \rightarrow \operatorname{Ext}_{G}^{1}\left(L\left(p \omega_{\alpha}\right), Q\right) \rightarrow \cdots
\end{aligned}
$$

All the weights in $Q$ are strictly less than $p \omega_{\alpha}-\alpha$. Therefore, $\operatorname{Hom}_{G}\left(L\left(p \omega_{\alpha}\right), Q\right)=0$ and the desired isomorphism will follow if it can be shown that $\operatorname{Ext}_{G}^{1}\left(L\left(p \omega_{\alpha}\right), Q\right)=$ 0 . To show this vanishing, it suffices to show that $\operatorname{Ext}_{G}^{1}\left(L\left(p \omega_{\alpha}\right), L(\mu)\right)=0$ for all composition factors $L(\mu)$ of $Q$. For such a composition factor $L(\mu)$, define $N$ via the short exact sequence

$$
0 \rightarrow L(\mu) \rightarrow H^{0}(\mu) \rightarrow N \rightarrow 0
$$

and consider the long exact sequence

$$
\cdots \rightarrow \operatorname{Hom}_{G}\left(L\left(p \omega_{\alpha}\right), N\right) \rightarrow \operatorname{Ext}_{G}^{1}\left(L\left(p \omega_{\alpha}\right), L(\mu)\right) \rightarrow \operatorname{Ext}_{G}^{1}\left(L\left(p \omega_{\alpha}\right), H^{0}(\mu)\right) \cdots
$$

As before, we have $\operatorname{Hom}_{G}\left(L\left(p \omega_{\alpha}\right), N\right)=0$. Consequently, the map

$$
\operatorname{Ext}_{G}^{1}\left(L\left(p \omega_{\alpha}\right), L(\mu)\right) \rightarrow \operatorname{Ext}_{G}^{1}\left(L\left(p \omega_{\alpha}\right), H^{0}(\mu)\right)
$$

is an embedding. By Proposition 4.3(b), $\operatorname{Ext}_{G}^{1}\left(L\left(p \omega_{\alpha}\right), H^{0}(\mu)\right)=0$, unless $\mu=$ $p \omega_{\alpha}-\alpha$ or $\mu=0$ and $\operatorname{Ext}_{G / G_{1}}^{1}\left(L\left(p \omega_{\alpha}\right), k\right) \neq 0$. But, the weight $\mu$ is strictly less than $p \omega_{\alpha}-\alpha$. Therefore, $\mu=0$. In order to have $\operatorname{Ext}_{G / G_{1}}^{1}\left(L\left(p \omega_{\alpha}\right), k\right) \cong$ $\operatorname{Ext}_{G}^{1}\left(L\left(\omega_{\alpha}\right), k\right) \neq 0$, the weights $\omega_{\alpha}$ and zero have to be linked. So there must exist a weight $\nu$ and an element $w$ of the Weyl group such that $\omega_{\alpha}=p \nu+w \cdot 0=$ $p \nu+w(\rho)-\rho$. Solving this equation for $p \nu$ and taking the inner product with a simple root $\beta \neq \alpha$ yields $p\left\langle\nu, \beta^{\vee}\right\rangle=1+\left\langle-w(\rho), \beta^{\vee}\right\rangle \leq h$. This forces $\left\langle\nu, \beta^{\vee}\right\rangle=0$ and therefore $\nu=\omega_{\alpha}$ and $(p-1) \omega_{\alpha}=\rho-w(\rho)$. This time we take the inner product with $\alpha_{0}$ and obtain $(p-1)\left\langle\omega_{\alpha}, \alpha_{0}^{\vee}\right\rangle=h-1+\left\langle-w(\rho), \alpha_{0}^{\vee}\right\rangle \leq 2(h-1)$. Since $p>h$ it follows that $\omega_{\alpha}$ is a minuscule weight and contained in the bottom alcove. (Note that this conclusion clearly holds if $\Phi$ is of type $A_{1}$.) Thus, $\operatorname{Ext}_{G}^{1}\left(L\left(\omega_{\alpha}\right), k\right)=0$ and also $\operatorname{Ext}_{G}^{1}\left(L\left(p \omega_{\alpha}\right), Q\right)=0$. 
4.6. In Corollary 4.2(B), we have shown that $\mathrm{H}^{1}\left(G\left(\mathbb{F}_{q}\right), L(\mu)\right) \cong \mathrm{H}^{1}(G, L(\mu))$ for weights $\mu \in X_{r}(T)$ that are $G_{1}$-linked to the zero weight. The following theorem provides necessary conditions for weights $\mu \in X_{r}(T)$, not $G_{1}$-linked to the zero weight, so that $\mathrm{H}^{1}\left(G\left(\mathbb{F}_{q}\right), L(\mu)\right) \neq 0$. Clearly, for such weights we always have $\mathrm{H}^{1}(G, L(\mu))=0$.

Proposition. Let $G$ be a simple simply connected algebraic group with $p \geq 3(h-1)$, and let $\mu \in X_{r}(T)$ not be $G_{1}$-linked to the zero weight.

(a) If $\mathrm{H}^{1}\left(G\left(\mathbb{F}_{p}\right), L(\mu)\right) \neq 0$ and $r=1$, then $\mu=p \rho_{w}+w \cdot\left(-w_{0} \nu\right)$ for some $w \in W \backslash\{1\}$ and some $0 \neq \nu \leq \rho_{w}$.

(b) If $\mathrm{H}^{1}\left(G\left(\mathbb{F}_{q}\right), L(\mu)\right) \neq 0$ and $r \geq 2$, then $\mu=p^{r-1}\left(p \rho_{w}+w \cdot 0\right)-w_{0} \nu$ for some $w \in W \backslash\{1\}$ and some $0 \neq \nu \leq \rho_{w}$.

Proof. (a) Assume that $\mathrm{H}^{1}\left(G\left(\mathbb{F}_{p}\right), L(\mu)\right) \neq 0$. According to the remark following BNP1 Cor. 7.4], there exists some $-w_{0} \nu \in \Gamma$ such that $\mathrm{H}^{1}\left(G, T_{-w_{0} \nu}^{0}(L(\mu) \otimes\right.$ $\left.\left.L\left(-w_{0} \nu\right)^{(1)}\right)\right) \neq 0$. In particular, the weight $\mu-p w_{0} \nu$ has to be linked to $-w_{0} \nu$ and $-w_{0} \nu \neq 0$. Thus $\mu=p \rho_{w}+w \cdot\left(-w_{0} \nu\right)$ for some $w \in W$. It remains to show that $\nu \leq \rho_{w}$. If $p \nu \leq p \rho_{w}+w \cdot 0$, since $p \rho_{w}+w \cdot 0 \leq p \rho_{w}$, we immediately conclude that $\nu \leq \rho_{w}$.

Suppose now that $p \nu \not \leq p \rho_{w}+w \cdot 0$. We have

$$
\begin{aligned}
T_{-w_{0} \nu}^{0}\left(L(\mu) \otimes L\left(-w_{0} \nu\right)^{(1)}\right) & =T_{-w_{0} \nu}^{0}\left(L\left(p \rho_{w}+w \cdot\left(-w_{0} \nu\right)\right) \otimes L\left(-w_{0} \nu\right)^{(1)}\right) \\
& \cong L\left(p \rho_{w}+w \cdot 0\right) \otimes L\left(-w_{0} \nu\right)^{(1)} .
\end{aligned}
$$

Therefore, $\operatorname{Ext}_{G}^{1}\left(L(\nu)^{(1)}, L\left(p \rho_{w}+w \cdot 0\right)\right) \neq 0$. Consider the short exact sequence

$$
0 \rightarrow L\left(p \rho_{w}+w \cdot 0\right) \rightarrow H^{0}\left(p \rho_{w}+w \cdot 0\right) \rightarrow Q \rightarrow 0
$$

and the associated long exact sequence

$$
\begin{aligned}
\cdots & \rightarrow \operatorname{Hom}_{G}(L(p \nu), Q) \rightarrow \operatorname{Ext}_{G}^{1}\left(L(p \nu), L\left(p \rho_{w}+w \cdot 0\right)\right) \\
& \rightarrow \operatorname{Ext}_{G}^{1}\left(L(p \nu), H^{0}\left(p \rho_{w}+w \cdot 0\right)\right) \rightarrow \cdots .
\end{aligned}
$$

Since the composition factors of $Q$ all have highest weight less than $p \rho_{w}+w \cdot 0$, $\operatorname{Hom}_{G}(L(p \nu), Q)=0$ and there is an embedding

$$
\operatorname{Ext}_{G}^{1}\left(L(p \nu), L\left(p \rho_{w}+w \cdot 0\right)\right) \hookrightarrow \operatorname{Ext}_{G}^{1}\left(L(p \nu), H^{0}\left(p \rho_{w}+w \cdot 0\right)\right) .
$$

Hence the latter Ext-group is also nonzero. By Proposition 4.3(b), we must have $\nu=\omega_{\alpha}$ and $p \rho_{w}+w \cdot 0=p \omega_{\alpha}-\alpha$ for some simple root $\alpha$. Hence $\rho_{w}=\omega_{\alpha}=\nu$.

(b) Assume that $\mathrm{H}^{1}\left(G\left(\mathbb{F}_{q}\right), L(\mu)\right) \neq 0$ and that $\mu$ is not $G_{1}$-linked to the zero weight. We apply Theorem 2.4(b) with $\lambda=0$. It follows that there exists a weight $\nu \in \Gamma$ such that $\operatorname{Ext}_{G}^{1}\left(L(\nu)^{(1)}, L\left(\mu_{r-1}\right)\right) \neq 0, \mu_{0}=-w_{0} \nu$, and $\mu_{i}=0$ for $i=1, \ldots, r-2$. Now $\mu_{r-1}$ has to be $G_{1}$-linked to zero. Thus, $\mu_{r-1}=p \rho_{w}+w \cdot 0$ for some $w \in W$. The argument in part (a) shows that $\nu \leq \rho_{w}$ and $w \neq 1$.

It is a consequence of Section 3 that for $r \geq 2$, the first $G\left(\mathbb{F}_{q}\right)$-cohomology of any simple module can be obtained from the first $G$-cohomology for $p^{r}$-bounded simple modules. The following corollary shows that the two are essentially the same.

Corollary. Let $G$ be a simple simply connected algebraic group with $p \geq 3(h-1)$. Suppose $r \geq 2$ and $\mu, \nu \in X_{r}(T)$ such that $\mu+p^{r} \nu \in \pi_{1, r}$. If $\mu$ is $G_{1}$-linked to the zero weight, then the restriction map

$$
\mathrm{H}^{1}\left(G, L\left(\mu+p^{r} \nu\right)\right) \rightarrow \mathrm{H}^{1}\left(G\left(\mathbb{F}_{q}\right), L\left(\mu+p^{r} \nu\right)\right) \cong \mathrm{H}^{1}\left(G\left(\mathbb{F}_{q}\right), L(\mu+\nu)\right)
$$


is an isomorphism. Moreover, all the nonzero first cohomology for simple modules of the finite group can be obtained in this fashion.

Proof. For $p=3$ and $G$ of type $A_{1}$, the assertion follows from [AJL. For the remaining cases, we will first show that the restriction map is an injection. For $\nu=0$, it follows from Corollary 4.2(B) that the map is an isomorphism. Assume that $\nu \neq 0$ and that $\mathrm{H}^{1}\left(G, L\left(\mu+p^{r} \nu\right)\right) \neq 0$. We apply Theorem 2.4(a) with $\lambda=0$ and obtain that for $0 \leq i \leq r-2$, the $\mu_{i}$ in the $p$-adic expansion of $\mu$ are zero. Moreover, it follows that $\mathrm{H}^{1}\left(G, L\left(\mu+p^{r} \nu\right)\right) \cong \mathrm{H}^{1}\left(G, L\left(\mu_{r-1}+p \nu\right)\right)$. Since $\mu_{r-1}+p \nu$ is $p^{r}$-restricted we have, again using Corollary $4.2(\mathrm{~B})$, that $\mathrm{H}^{1}\left(G, L\left(\mu_{r-1}+p \nu\right)\right) \cong$ $\mathrm{H}^{1}\left(G\left(\mathbb{F}_{q}\right), L\left(\mu_{r-1}+p \nu\right)\right)$. Now let $E$ be a nontrivial extension of the trivial module by $L\left(\mu+p^{r} \nu\right)$. Then $E \cong \widetilde{E}^{(r-1)}$ where $\widetilde{E}$ is a nontrivial extension of the trivial module by $L\left(\mu_{r-1}+p \nu\right)$. Now the Frobenius map is an automorphism of $G\left(\mathbb{F}_{q}\right)$. Therefore $E \cong \widetilde{E}^{(r-1)}$ remains nonsplit for $G\left(\mathbb{F}_{q}\right)$. Finally, $L\left(\mu+p^{r} \nu\right) \cong L(\mu+\nu)$ as a $G\left(\mathbb{F}_{q}\right)$-module.

It remains to be shown that all nontrivial extensions of the trivial module by a simple $G\left(\mathbb{F}_{q}\right)$-module can be obtained in this fashion. Let $\lambda \in X_{r}(T)$ with $\mathrm{H}^{1}\left(G\left(\mathbb{F}_{q}\right), L(\lambda)\right) \neq 0$. If $\lambda$ is $G_{1}$-linked to zero, then Corollary $4.2(\mathrm{~B})$ says that $\mathrm{H}^{1}\left(G\left(\mathbb{F}_{q}\right), L(\lambda)\right) \cong \mathrm{H}^{1}(G, L(\lambda))$. Now assume that $\lambda$ is not $G_{1}$-linked to the zero weight. According to the previous Proposition, there exists a $w \in W \backslash\{1\}$ and a $\nu \in \Gamma$ such that $\lambda=p^{r-1}\left(p \rho_{w}+w \cdot 0\right)+\nu$. Set $\mu=p^{r-1}\left(p \rho_{w}+w \cdot 0\right)$. As we did above, we use the formulas in Theorem 2.4(b) to show that

$$
\operatorname{Ext}_{G\left(\mathbb{F}_{q}\right)}^{1}(k, L(\lambda))=\operatorname{Ext}_{G\left(\mathbb{F}_{q}\right)}^{1}(k, L(\mu+\nu)) \cong \operatorname{Ext}_{G}^{1}\left(k, L\left(p^{-(r-1)} \mu+p \nu\right)\right) .
$$

Similarly, Theorem 2.4(a) implies that

$$
\operatorname{Ext}_{G}^{1}\left(k, L\left(\mu+p^{r} \nu\right)\right) \cong \operatorname{Ext}_{G}^{1}\left(k, L\left(p^{-(r-1)} \mu+p \nu\right)\right) \text {. }
$$

4.7. The next theorem shows that there exists nonvanishing of $G\left(\mathbb{F}_{q}\right)$-cohomology for simple modules with vanishing $G$-cohomology.

Theorem. Let $G$ be a simple simply connected algebraic group with $p \geq 3(h-1)$. Let $\lambda \in X_{r}(T)$.

(a) For $r=1$ and any simple root $\alpha$,

$$
\mathrm{H}^{1}\left(G\left(\mathbb{F}_{p}\right), L\left(p \omega_{\alpha}+s_{\alpha} \cdot\left(-w_{0} \omega_{\alpha}\right)\right)\right) \cong k .
$$

(b) For $r \geq 2$ and any simple root $\alpha$,

$$
\mathrm{H}^{1}\left(G\left(\mathbb{F}_{q}\right), L\left(p^{r} \omega_{\alpha}-p^{r-1} \alpha-w_{0} \omega_{\alpha}\right)\right) \cong k .
$$

Proof. (a) We start with the remark following [BNP1, Cor. 7.4] and look for all $\nu \in \Gamma$ such that $\mathrm{H}^{1}\left(G, T_{\nu}^{0}\left(L\left(p \omega_{\alpha}+s_{\alpha} \cdot\left(-w_{0} \omega_{\alpha}\right)\right) \otimes L(\nu)^{(1)}\right)\right) \neq 0$. Any such $\nu$ has to be $G_{1}$-linked to $-w_{0} \omega_{\alpha}$. It follows from Jan2, Lem. 1] that there exists a $w \in W$ and its corresponding $\rho_{w}$ such that $-w_{0} \omega_{\alpha}=w \cdot \nu+p \rho_{w}$. We rewrite this expression as $p \rho_{w}=-w_{0} \omega_{\alpha}-w(\nu+\rho)+\rho$. Taking the absolute value of the inner product of the right-hand side with any simple root $\beta$ results in an integer less than or equal to $2(h-1)+2$, which is less than $p$. The inner product of the left-hand side is divisible by $p$. Therefore, both sides have to be zero. This forces $w=1$ and $\nu=-w_{0} \omega_{\alpha}$. Using the identity

$$
T_{-w_{0} \omega_{\alpha}}^{0}\left(L\left(p \omega_{\alpha}+s_{\alpha} \cdot\left(-w_{0} \omega_{\alpha}\right)\right) \otimes L\left(w_{0} \omega_{\alpha}\right)^{(1)}\right) \cong L\left(p \omega_{\alpha}-\alpha\right) \otimes L\left(-w_{0} \omega_{\alpha}\right)^{(1)}
$$


and Proposition 4.5 we obtain

$$
\begin{aligned}
\mathrm{H}^{1}(G & \left.\left(\mathbb{F}_{p}\right), L\left(p \omega_{\alpha}+s_{\alpha} \cdot\left(-w_{0} \omega_{\alpha}\right)\right)\right) \\
& \cong \mathrm{H}^{1}\left(G, T_{-w_{0} \omega_{\alpha}}^{0}\left(L\left(p \omega_{\alpha}+s_{\alpha} \cdot\left(-w_{0} \omega_{\alpha}\right)\right) \otimes L\left(-w_{0} \omega_{\alpha}\right)^{(1)}\right)\right) \\
& \cong \mathrm{H}^{1}\left(G, L\left(p \omega_{\alpha}-\alpha\right) \otimes L\left(-w_{0} \omega_{\alpha}\right)^{(1)}\right) \\
& \cong \operatorname{Ext}_{G}^{1}\left(L\left(\omega_{\alpha}\right)^{(1)}, L\left(p \omega_{\alpha}-\alpha\right)\right) \\
& \cong k
\end{aligned}
$$

(b) According to Corollary 4.6,

$$
\begin{aligned}
\mathrm{H}^{1}(G & \left.\left(\mathbb{F}_{q}\right), L\left(p^{r} \omega_{\alpha}-p^{r-1} \alpha-w_{0} \omega_{\alpha}\right)\right) \\
& \cong \mathrm{H}^{1}\left(G, L\left(p^{r} \omega_{\alpha}-p^{r-1} \alpha-p^{r} w_{0} \omega_{\alpha}\right)\right) \\
& \cong \mathrm{H}^{1}\left(G, L\left(p^{r} \omega_{\alpha}-p^{r-1} \alpha\right) \otimes L\left(-w_{0} \omega_{\alpha}\right)^{(r)}\right) \\
& \cong \operatorname{Ext}_{G}^{1}\left(L\left(\omega_{\alpha}\right)^{(r)}, L\left(p^{r} \omega_{\alpha}-p^{r-1} \alpha\right)\right) \\
& \cong \operatorname{Ext}_{G}^{1}\left(L\left(\omega_{\alpha}\right)^{(1)}, L\left(p \omega_{\alpha}-\alpha\right)\right) \\
& \cong k
\end{aligned}
$$

The last isomorphism follows from Proposition 4.5.

4.8. Jantzen [Jan6, Prop. 2.2] shows that for any prime and any $G$-module $V$ whose composition factors have highest weights $\mu$ satisfying

$$
\left\langle\mu, \alpha_{0}^{\vee}\right\rangle \leq \begin{cases}p^{r}-3 p^{r-1}-3 & \text { for } \Phi \text { of type } G_{2}, \\ p^{r}-2 p^{r-1}-2 & \text { otherwise }\end{cases}
$$

the restriction map $\mathrm{H}^{1}(G, V) \rightarrow \mathrm{H}^{1}\left(G\left(\mathbb{F}_{q}\right), V\right)$ is an isomorphism. His analysis uses results of CPSK.

Andersen [And2 Thm. 2.8] showed that for primes $p \geq 3(h-1)$, a better bound can be obtained for certain $G$-modules $V$. If the composition factors have highest weights $\mu$ satisfying

$$
\left\langle\mu, \alpha_{0}^{\vee}\right\rangle \leq p^{r}-p^{r-1}-2 \text { and } \Phi \text { is not of type } A_{1},
$$

then the restriction map $\mathrm{H}^{1}(G, V) \rightarrow \mathrm{H}^{1}\left(G\left(\mathbb{F}_{q}\right), V\right)$ is an isomorphism. For a generalization of [And2, Thm. 2.8], see Corollary 5.4(B) part (b).

Similar results were also obtained by Friedlander and Parshall in $[\mathrm{FP}]$ and $[\mathrm{F}]$. Moreover, their work extends to the higher cohomology groups. Here we restrict ourselves to the first cohomology. With the help of Theorem 4.7, we obtain the best possible bounds to insure that $\mathrm{H}^{1}(G, V) \rightarrow \mathrm{H}^{1}\left(G\left(\mathbb{F}_{q}\right), V\right)$ is an isomorphism. These are the optimal bounds because Theorem 4.7 can be used to produce counterexamples to any lower bound.

Theorem (A). Let $G$ be a simple simply connected algebraic group with $p \geq 3(h-1)$ and let $r \geq 2$. For any $G$-module $V$ whose composition factors have highest weights $\mu$ satisfying

$$
\left\langle\mu, \alpha_{0}^{\vee}\right\rangle \leq \begin{cases}p^{r}-2 p^{r-1} & \text { for } \Phi \text { of type } A_{1}, \\ p^{r}-p^{r-1} & \text { for } \Phi \text { of type } A_{n}, n \geq 2, \\ p^{r} & \text { for } \Phi \text { of type } B_{n}, C_{n} \text { for } n \geq 2, D_{n}, E_{6}, E_{7}, \\ 2 p^{r}-p^{r-1}+1 & \text { for } \Phi \text { of type } G_{2}, F_{4}, E_{8},\end{cases}
$$


the restriction map $\mathrm{H}^{1}(G, V) \rightarrow \mathrm{H}^{1}\left(G\left(\mathbb{F}_{q}\right), V\right)$ is an isomorphism.

Proof. Without loss of generality, we may assume that $V$ is indecomposable as a $G$-module. Moreover, we will first assume that all simple $G$-composition factors of $V$ have $p^{r}$-restricted highest weights.

If these highest weights are $G_{1}$-linked to the zero weight, the result follows immediately from Corollary 4.2(A) or AJL when $p=3$ and the root system is of type $A_{1}$. If they are not $G_{1}$-linked to the zero weight, then $\mathrm{H}^{1}(G, V)=0$. It is now sufficient to show that $\mathrm{H}^{1}\left(G\left(\mathbb{F}_{q}\right), L(\mu)\right)=0$ for any composition factor $L(\mu)$ of $V$. We proceed by identifying the weights $\lambda$, not $G_{1}$-linked to the zero weight, with $\mathrm{H}^{1}\left(G\left(\mathbb{F}_{q}\right), L(\lambda)\right) \neq 0$ and $\left\langle\lambda, \alpha_{0}^{\vee}\right\rangle$ minimal. By Proposition 4.6(b), $\lambda=p^{r-1}\left(p \rho_{w}+w \cdot 0\right)-w_{0} \nu$ for some $w \in W$ and some $0 \neq \nu \leq \rho_{w}$. Clearly for such a $\lambda$ we need $\left\langle\rho_{w}, \alpha_{0}^{\vee}\right\rangle$ to be minimal. For types $A_{n}, B_{n}, C_{n}, D_{n}, E_{6}$, and $E_{7}$, we can find minuscule fundamental weights $\omega_{\alpha}$ with $\left\langle\omega_{\alpha}, \alpha_{0}^{\vee}\right\rangle=1$. For types $G_{2}, F_{4}$, and $E_{8}$, the smallest fundamental weight satisfies $\left\langle\omega_{\alpha}, \alpha_{0}^{v}\right\rangle=2$. In both cases, the condition $0 \neq \nu \leq \omega_{\alpha}$ forces $\nu=\omega_{\alpha}$. According to Corollary 4.6,

$$
\begin{aligned}
\mathrm{H}^{1}\left(G\left(\mathbb{F}_{q}\right), L(\lambda)\right) & =\mathrm{H}^{1}\left(G\left(\mathbb{F}_{q}\right), L\left(p^{r} \omega_{\alpha}+p^{r-1} w \cdot 0-w_{0} \omega_{\alpha}\right)\right) \\
& \cong \mathrm{H}^{1}\left(G, L\left(p^{r} \omega_{\alpha}+p^{r-1} w \cdot 0-p^{r} w_{0} \omega_{\alpha}\right)\right) \\
& \cong \mathrm{H}^{1}\left(G, L\left(p^{r} \omega_{\alpha}+p^{r-1} w \cdot 0\right) \otimes L\left(-w_{0} \omega_{\alpha}\right)^{(r)}\right) \\
& \cong \operatorname{Ext}_{G}^{1}\left(L\left(\omega_{\alpha}\right)^{(r)}, L\left(p^{r} \omega_{\alpha}+p^{r-1} w \cdot 0\right)\right) \\
& \cong \operatorname{Ext}_{G}^{1}\left(L\left(\omega_{\alpha}\right)^{(1)}, L\left(p \omega_{\alpha}+w \cdot 0\right)\right) .
\end{aligned}
$$

A closer look at the proof of Proposition 4.5 shows that the last term is zero unless $w=s_{\alpha}$. This implies that $\lambda=p^{r} \omega_{\alpha}-p^{r-1} \alpha-w_{0} \omega_{\alpha}$. Checking all possible minimal weights $\omega_{\alpha}$ and their corresponding roots $\alpha$ shows that $\left\langle\alpha, \alpha_{0}^{\vee}\right\rangle=2$ for type $A_{1},\left\langle\alpha, \alpha_{0}^{\vee}\right\rangle=1$ for types $A_{n}(n \geq 2), G_{2}, F_{4}$, and $E_{8}$, and $\left\langle\alpha, \alpha_{0}^{\vee}\right\rangle=$ 0 for types $B_{n}, C_{n}, D_{n}, E_{6}$, and $E_{7}$. Therefore, the "smallest" weights $\lambda$ with $\mathrm{H}^{1}\left(G\left(\mathbb{F}_{q}\right), L(\lambda)\right) \neq 0$ are just outside the allowed region. Hence, $\mathrm{H}^{1}\left(G\left(\mathbb{F}_{q}\right), V\right)=$ $0=\mathrm{H}^{1}(G, V)$ and the bounds are the best possible.

For types $B_{n}, C_{n}, D_{n}, E_{6}$, and $E_{7}$, our bound on the weights allows for modules $V$ that contain simple composition factors whose highest weights are not $p^{r}$-restricted. However, the given bound on the weights allows only factors that are isomorphic to $L\left(p^{r} \omega_{\alpha}\right)$, where $\omega_{\alpha}$ is a minuscule weight. For root systems of the above type, the minuscule weights are not contained in the root lattice. Thus $p^{r} \omega_{\alpha}$ is not $G_{1}$-linked to the zero weight and $\mathrm{H}^{1}\left(G, L\left(p^{r} \omega_{\alpha}\right)\right)=0$. Further, if $V$ contains such a composition factor, then $\mathrm{H}^{1}(G, V)=0$ since none of the composition factors would be $G_{1}$-linked to zero. Therefore it is sufficient to show that $\mathrm{H}^{1}\left(G\left(\mathbb{F}_{q}\right), L(\mu)\right)=0$ for any composition factor $L(\mu)$ of $V$. If $\mu$ is restricted, this was shown above, and for unrestricted, by Proposition 4.6(b), we have $\mathrm{H}^{1}\left(G\left(\mathbb{F}_{q}\right), L\left(p^{r} \omega_{\alpha}\right)\right) \cong \mathrm{H}^{1}\left(G\left(\mathbb{F}_{q}\right), L\left(\omega_{\alpha}\right)\right)=0$.

In the case $r=1$, we can make the following statements about the vanishing of cohomology.

Theorem (B). Let $G$ be a simple simply connected algebraic group with $p \geq 3(h-1)$ $\left(p>3\right.$ for type $\left.A_{1}\right)$. For any $G$-module $V$ whose composition factors have highest 
weights $\mu$ satisfying

$$
\begin{aligned}
& \left\langle\mu, \alpha_{0}^{\vee}\right\rangle \leq \begin{cases}p-4 & \text { for } \Phi \text { of type } A_{1}, \\
p-1 & \text { for } \Phi \text { of type } A_{n}, n \geq 2, \\
p & \text { for } \Phi \text { of type } B_{n}, C_{n} \text { for } n \geq 2, D_{n}, E_{6}, E_{7}, \\
2(p-h)+1 & \text { for } \Phi \text { of type } G_{2}, F_{4}, E_{8},\end{cases} \\
& \mathrm{H}^{1}\left(G\left(\mathbb{F}_{p}\right), V\right) \cong \mathrm{H}^{1}(G, V)=0 .
\end{aligned}
$$

Proof. The argument is almost identical to the previous proof. The smallest nonzero dominant weight $\lambda$ that is linked to the zero weight satisfies $\left\langle\lambda, \alpha_{0}^{\vee}\right\rangle=2(p-(h-1))$. Hence $\mathrm{H}^{1}(G, V)=0$. It is sufficient to show that $\mathrm{H}^{1}\left(G\left(\mathbb{F}_{p}\right), L(\mu)\right)=0$ for any weight $\mu$ satisfying the given bounds. If $\mu$ is $p$-restricted and $G_{1}$-linked to the zero weight, the result follows from Corollary $4.2(\mathrm{~B})$.

We proceed by identifying the $p$-restricted weights $\lambda$, not $G_{1}$-linked to the zero weight, with $\mathrm{H}^{1}\left(G\left(\mathbb{F}_{p}\right), L(\lambda)\right) \neq 0$ and $\left\langle\lambda, \alpha_{0}^{\vee}\right\rangle$ minimal. We apply Proposition 4.6(a) and find that $\lambda=p \rho_{w}+w \cdot\left(-w_{0} \nu\right)$, for some $w \in W$ and some $0 \neq \nu \leq \rho_{w}$. Clearly for such a $\lambda$ we need $\left\langle\rho_{w}, \alpha_{0}^{\vee}\right\rangle$ to be minimal. For types $A_{n}, B_{n}, C_{n}, D_{n}, E_{6}$, and $E_{7}$ we can find minuscule fundamental weights $\omega_{\alpha}$ with $\left\langle\omega_{\alpha}, \alpha_{0}^{\vee}\right\rangle=1$. For types $G_{2}, F_{4}$, and $E_{8}$, the smallest fundamental weight satisfies $\left\langle\omega_{\alpha}, \alpha_{0}^{\vee}\right\rangle=2$. In both cases, the condition $0 \neq \nu \leq \omega_{\alpha}$ forces $\nu=\omega_{\alpha}$. As in the proof of Theorem 4.8(A), we find that for a "minimal" $\lambda$ we have $w=s_{\alpha}$ and $\lambda=p \omega_{\alpha}+s_{\alpha} \cdot\left(-w_{0} \omega_{\alpha}\right)$. For types $A_{n}, B_{n}, C_{n}, D_{n}, E_{6}$, and $E_{7}$ one checks all minuscule weights and their corresponding roots $\alpha$ to find the "minimal" $\lambda$. The bounds given in the Proposition were chosen accordingly. For types $G_{2}, F_{4}$ and $E_{8}$ the smallest $\lambda$ not linked to the zero weight is larger than the smallest $\lambda \neq 0$ that is linked to the zero weight. Therefore, the algebraic group provides the "minimal" weight for these types.

Finally, we have to deal with the nonrestricted cases. Again the only possibilities are modules isomorphic to $L\left(p \omega_{\alpha}\right)$, where $\omega_{\alpha}$ is a minuscule weight for types $B_{n}, C_{n}, D_{n}, E_{6}$, and $E_{7}$. But $L\left(p \omega_{\alpha}\right) \cong L\left(\omega_{\alpha}\right)$ as a $G\left(\mathbb{F}_{p}\right)$-module and our previous argument settles this case.

4.9. As an immediate consequence of our results, we recover the following known facts.

Corollary. Let $G$ be a simple simply connected algebraic group with $p \geq 3(h-1)$.

(a) If $r \geq 2$ and $\mu \in X_{r-1}(T)$, then $\mathrm{H}^{1}\left(G\left(\mathbb{F}_{q}\right), L(\mu)\right) \cong \mathrm{H}^{1}(G, L(\mu))$.

(b) If $\mu \in \bar{C}_{\mathbb{Z}}$ and the underlying root system is not of type $A_{1}$, then $\mathrm{H}^{1}\left(G\left(\mathbb{F}_{q}\right)\right.$, $L(\mu))=0$.

(c) If $\omega$ is a fundamental weight, then $\mathrm{H}^{1}\left(G\left(\mathbb{F}_{q}\right), L(\omega)\right)=0$.

4.10. In the previous sections, we provided conditions under which $\mathrm{H}^{1}\left(G\left(\mathbb{F}_{q}\right)\right.$, $L(\mu)) \cong \mathrm{H}^{1}(G, L(\mu))$. We conclude this section with some sufficient conditions on $\mu \in X_{r}(T)$ so that $\mathrm{H}^{1}\left(G_{r}, L(\mu)\right) \cong \mathrm{H}^{1}(G, L(\mu))$. See [Jan5] for explicit descriptions of $\mathrm{H}^{1}\left(G_{1}, L(\mu)\right)$. Here and in parts of Section 5 , we make use of the strong linkage principle Jan1 II.6.13 and replace the order relation " $\leq$ " on the weights by the refined order relation " $\uparrow$ " as defined in [Jan1, II.6.4].

Theorem. Let $G$ be a simple simply connected algebraic group, $p \geq 3(h-1)$, and $\mu \in X_{r}(T)$. If

(a) $p^{r} \nu \not X \mu$ when $\nu \in X(T)_{+}-\{0\}$, and 
(b) $\mu \neq p^{r} \omega_{\alpha}-p^{r-1} \alpha$ for some simple root $\alpha$, then $\mathrm{H}^{1}\left(G_{r}, L(\mu)\right) \cong \mathrm{H}^{1}(G, L(\mu))$.

Proof. If $\dot{\mu} \neq 0$, the claim follows immediately from Corollary 2.7(A). On the other hand, suppose $\dot{\mu}=0$. Then $\mu=p^{r-1} \mu_{r-1}$ and

$$
\mathrm{H}^{1}\left(G_{r}, L(\mu)\right)=\mathrm{H}^{1}\left(G_{r}, L\left(\mu_{r-1}\right)^{(r-1)} \cong \mathrm{H}^{1}\left(G_{1}, L\left(\mu_{r-1}\right)\right)^{(r-1)} .\right.
$$

Condition (a) implies that $p \nu \chi \mu_{r-1}$, while condition (b) implies that $\mu_{r-1} \neq$ $p \omega_{\alpha}-\alpha$ for any simple root $\alpha$. Hence, by And2, $\mathrm{H}^{1}\left(G_{1}, H^{0}\left(\mu_{r-1}\right)\right)=0$ and we may apply Lemma 5.1 to conclude that $\mathrm{H}^{1}\left(G_{1}, L\left(\mu_{r-1}\right)\right) \cong \mathrm{H}^{1}\left(G, L\left(\mu_{r-1}\right)\right)$. Finally, $\mathrm{H}^{1}(G, L(\mu))=\mathrm{H}^{1}\left(G, L\left(\mu_{r-1}\right)^{(r-1)}\right) \cong \mathrm{H}^{1}\left(G, L\left(\mu_{r-1}\right)\right)$.

We now present some consequences of Theorem 4.10.

Corollary. Let $G$ be a simple simply connected algebraic group, $p \geq 3(h-1)$, and $\mu \in X_{r}(T)$. If

(a) $\left\langle\mu_{r-1}, \alpha_{0}^{\vee}\right\rangle \leq p-1$, and

(b) $\left\langle\mu_{r-1}, \alpha^{\vee}\right\rangle \neq p-2$ for all simple roots $\alpha$, then $\mathrm{H}^{1}\left(G_{r}, L(\mu)\right) \cong \mathrm{H}^{1}(G, L(\mu))$.

Proof. For any $\nu \in X(T)_{+}-\{0\}$, if condition (a) holds, then $\left\langle p \nu, \alpha_{0}^{\vee}\right\rangle \geq p>p-1 \geq$ $\left\langle\mu_{r-1}, \alpha_{0}^{\vee}\right\rangle$, which implies that $p \nu$ is not less than or equal to $\mu_{r-1}$ and hence that $p \nu \ngtr \mu_{r-1}$. Further, condition (b) implies that $\mu_{r-1} \neq p \omega_{\alpha}-\alpha$ for any simple root $\alpha$. With these two facts, the claim follows as in the proof of the previous Theorem.

\section{General comparison results for extensions}

5.1. In this section we determine conditions under which $\operatorname{Ext}_{G\left(\mathbb{F}_{q}\right)}^{1}(L(\lambda), L(\mu))$ or $\operatorname{Ext}_{G_{r}}^{1}(L(\lambda), L(\mu))$ may be identified with $\operatorname{Ext}_{G}^{1}(L(\lambda), L(\mu))$. We begin with some preliminary results.

Lemma. Let $G$ be a simple algebraic group, $p>2$, and $\lambda, \mu \in X_{r}(T)$. If $\lambda+p^{r} \nu \not \gamma \mu$ and $\mu+p^{r} \nu \not \gamma \lambda$ for any $\nu \in X(T)_{+}-\{0\}$ and $\operatorname{Ext}_{G_{r}}^{1}\left(V(\lambda), H^{0}(\mu)\right)=0$, then

$$
\operatorname{Ext}_{G_{r}}^{1}(L(\lambda), L(\mu)) \cong \operatorname{Ext}_{G}^{1}(L(\lambda), L(\mu)) \text {. }
$$

Proof. By [And1, Thm. 4.5], one has $\operatorname{Ext}_{G_{r}}^{1}(L(\lambda), L(\lambda)) \cong \operatorname{Ext}_{G}^{1}(L(\lambda), L(\lambda))=$ 0 . Thus we may assume that $\lambda \neq \mu$. Furthermore, since $\operatorname{Ext}_{G_{r}}^{1}(L(\lambda), L(\mu)) \cong$ $\operatorname{Ext}_{G_{r}}^{1}(L(\mu), L(\lambda))$ and $\operatorname{Ext}_{G_{r}}^{1}\left(V(\lambda), H^{0}(\mu)\right) \cong \operatorname{Ext}_{G_{r}}^{1}\left(V(\mu), H^{0}(\lambda)\right)$ [Jan1. II.2.12(3), II.2.13(2)], we may assume, without loss of generality, that $\lambda \nmid \mu$.

There exists a short exact sequence of the form

$$
0 \rightarrow L(\mu) \rightarrow H^{0}(\mu) \rightarrow Q \rightarrow 0 .
$$

This induces a long exact sequence

$$
\cdots \rightarrow \operatorname{Hom}_{G_{r}}(L(\lambda), Q) \rightarrow \operatorname{Ext}_{G_{r}}^{1}(L(\lambda), L(\mu)) \rightarrow \operatorname{Ext}_{G_{r}}^{1}\left(L(\lambda), H^{0}(\mu)\right) \rightarrow \cdots .
$$

Observe that $\operatorname{Hom}_{G_{r}}(L(\lambda), Q)$ is a $G$-module and $\operatorname{Hom}_{G_{r}}(L(\lambda), Q) \otimes L(\lambda)$ is a $G$-submodule of $Q$ Jan3 2.2(1)]. Suppose that $p^{r} \nu$ is a highest weight of a composition factor of $\operatorname{Hom}_{G_{r}}(L(\lambda), Q)$. Then $\lambda+p^{r} \nu$ is a highest weight of a composition factor of $Q$ and of $H^{0}(\mu)$. Therefore, $\lambda+p^{r} \nu \uparrow \mu$. That forces $\nu=0$ and contradicts our assumption that $\lambda \Uparrow \mu$. Thus, $\operatorname{Hom}_{G_{r}}(L(\lambda), Q)=0$ and we obtain an embedding $\operatorname{Ext}_{G_{r}}^{1}(L(\lambda), L(\mu)) \hookrightarrow \operatorname{Ext}_{G_{r}}^{1}\left(L(\lambda), H^{0}(\mu)\right)$. Using $\operatorname{Ext}_{G_{r}}^{1}\left(L(\lambda), H^{0}(\mu)\right)$ 
$\cong \operatorname{Ext}_{G_{r}}^{1}(V(\mu), L(\lambda))$, we also obtain an embedding $\operatorname{Ext}_{G_{r}}^{1}(L(\mu), L(\lambda)) \hookrightarrow$ $\operatorname{Ext}_{G_{r}}^{1}(V(\mu), L(\lambda))$.

Consider now the short exact sequence

$$
0 \rightarrow L(\lambda) \rightarrow H^{0}(\lambda) \rightarrow N \rightarrow 0
$$

This induces a long exact sequence

$$
\begin{aligned}
\cdots \rightarrow \operatorname{Hom}_{G_{r}}\left(V(\mu), H^{0}(\lambda)\right) & \rightarrow \operatorname{Hom}_{G_{r}}(V(\mu), N) \\
& \rightarrow \operatorname{Ext}_{G_{r}}^{1}(V(\mu), L(\lambda)) \rightarrow 0 \rightarrow \cdots
\end{aligned}
$$

because $\operatorname{Ext}_{G_{r}}^{1}\left(V(\mu), H^{0}(\lambda)\right)$ is assumed to be zero. The $G_{r^{-}}$-socle of $H^{0}(\lambda)$ is $L(\lambda)$ because $\lambda$ is $p^{r}$-restricted. Moreover, $V(\mu)$ contains no dominant weights of the form $\lambda+p^{r} \nu$. Thus $\operatorname{Hom}_{G_{r}}\left(V(\mu), H^{0}(\lambda)\right) \cong 0$. We obtain an isomorphism of $G$ modules $\operatorname{Hom}_{G_{r}}(V(\mu), N) \cong \operatorname{Ext}_{G_{r}}^{1}(V(\mu), L(\lambda))$. Suppose that $p^{r} \nu$ is a nonzero highest weight of a composition factor of $\operatorname{Hom}_{G_{r}}(V(\mu), N)$. Then $L\left(\mu+p^{r} \nu\right)$ is a $G$-composition factor of $N$ because $V(\mu)$ has simple $G_{r}$-head $L(\mu)$. Thus $\mu+p^{r} \nu$ is a highest weight of a composition factor of $N$ and also of $H^{0}(\lambda)$. But this contradicts our assumption that $\mu+p^{r} \nu \chi \lambda$ for any nonzero dominant weight $\nu$. Since there are no self-extensions of the trivial module over $G, \operatorname{Hom}_{G_{r}}(V(\mu), N)$ is isomorphic to a direct sum of trivial modules. It follows that $\operatorname{Ext}_{G_{r}}^{1}(V(\mu), L(\lambda))$ and $\operatorname{Ext}_{G_{r}}^{1}(L(\mu), L(\lambda))$ are both direct sums of trivial modules.

Finally, consider the Lyndon-Hochschild-Serre spectral sequence for $G_{r} \unlhd G$ :

$$
E_{2}^{i, j}=\operatorname{Ext}_{G / G_{r}}^{i}\left(k, \operatorname{Ext}_{G_{r}}^{j}(L(\mu), L(\lambda))\right) \Rightarrow \operatorname{Ext}_{G}^{i+j}(L(\mu), L(\lambda)) .
$$

Since $\operatorname{Hom}_{G_{r}}(L(\mu), L(\lambda))=0$, we have $E_{2}^{1,0}=0=E_{2}^{2,0}$ and from the corresponding five-term exact sequence, $E^{1} \cong E_{2}^{0,1}$. Thus, since the $G$-structure on $\operatorname{Ext}_{G_{r}}^{1}(L(\mu)$, $L(\lambda))$ is trivial, one has

$$
\operatorname{Ext}_{G_{r}}^{1}(L(\mu), L(\lambda)) \cong \operatorname{Hom}_{G / G_{r}}\left(k, \operatorname{Ext}_{G_{r}}^{1}(L(\mu), L(\lambda))\right) \cong \operatorname{Ext}_{G}^{1}(L(\mu), L(\lambda)) .
$$

5.2. The methods used in the following proposition are similar to those in Sections 4.5 and 4.6 of BNP2.

Proposition. Let $G$ be a simple algebraic group and $\lambda, \mu \in X_{1}(T)$ with $\lambda \neq \mu$.

(a) If $p>3$ and $\left\langle\mu-\lambda, \alpha^{\vee}\right\rangle<p / 3$ for all roots $\alpha$, then one of the following holds:

(i) $\operatorname{Ext}_{G_{1}}^{1}\left(V(\lambda), H^{0}(\mu)\right)=0$.

(ii) the underlying root system is of type $A_{1}$ or $C_{n}$ and $\lambda=\mu+\left(\frac{p}{2}-\langle\mu+\right.$ $\left.\left.\rho, \alpha_{n}^{\vee}\right\rangle\right) \alpha_{n}$, where $\alpha_{n}$ is the unique long (last) simple root.

(b) If $p \geq h\left(p>3\right.$ for type $\left.A_{n}\right)$ and $\lambda=\mu+\alpha$ for some positive nonsimple root $\alpha$, then $\operatorname{Ext}_{G_{1}}^{1}\left(V(\lambda), H^{0}(\mu)\right)=0$.

Proof. The proof uses the same strategy as the proof of Proposition 4.6 in BNP2, although the details differ slightly.

(a) Assume that $\operatorname{Ext}_{G_{1}}^{1}\left(V(\lambda), H^{0}(\mu)\right) \cong \operatorname{Ext}_{G_{1}}^{1}\left(V(\mu), H^{0}(\lambda)\right) \neq 0$. Without loss of generality, we may assume that $\lambda \ngtr \mu$. The $G_{1}$-socle of $H^{0}(\lambda)$ is $L(\lambda)$ with $\lambda p$-restricted while $V(\mu)$ contains no dominant weights of the form $\lambda+p \xi$. It follows that $\operatorname{Hom}_{G_{1}}\left(V(\mu), H^{0}(\lambda)\right)=0$. Assume that $\operatorname{Ext}_{G_{1}}^{1}\left(V(\mu), H^{0}(\lambda)\right)$ has a composition factor $L(\nu)^{(1)}$ in its socle. Applying the five-term Lyndon-HochschildSerre sequence for $G_{1} \subset G$ (as in the proof of Proposition 4.3) yields an isomorphism

$$
0 \neq \operatorname{Hom}_{G / G_{1}}\left(L(\nu)^{(1)}, \operatorname{Ext}_{G_{1}}^{1}\left(V(\mu), H^{0}(\lambda)\right)\right) \cong \operatorname{Ext}_{G}^{1}\left(V(\mu) \otimes L(\nu)^{(1)}, H^{0}(\lambda)\right) .
$$


Since $\lambda$ is a dominant weight, it follows by Frobenius reciprocity that

$$
\begin{aligned}
0 \neq \operatorname{Ext}_{G}^{1}\left(V(\mu) \otimes L(\nu)^{(1)}, H^{0}(\lambda)\right) & \cong \operatorname{Ext}_{B}^{1}\left(V(\mu) \otimes L(\nu)^{(1)}, \lambda\right) \\
& \cong \operatorname{Ext}_{B}^{1}\left(V(\mu), \lambda \otimes L\left(-w_{0} \nu\right)^{(1)}\right) .
\end{aligned}
$$

Thus there exists a weight $\sigma$ such that $\left.\operatorname{Ext}_{B}^{1}(V(\mu), \lambda-p \sigma)\right) \neq 0$. It follows from [Jan1, II 4.13] that $\lambda-p \sigma \notin X(T)_{+}$. Using the spectral sequence [And1, 1.1]

$$
E_{2}^{i, j}=\operatorname{Ext}_{G}^{i}\left(V(\mu), R^{j} \operatorname{ind}_{B}^{G}(\lambda-p \sigma)\right) \Rightarrow \operatorname{Ext}_{B}^{i+j}(V(\mu), \lambda-p \sigma),
$$

we obtain the isomorphism

$$
\operatorname{Ext}_{B}^{1}(V(\mu), \lambda-p \sigma) \cong \operatorname{Hom}_{G}\left(V(\mu), H^{1}(\lambda-p \sigma)\right) .
$$

Now we apply [Jan1, II.5.4, II.5.15] and obtain two possibilities.

Case 1. There exists a dominant weight $\bar{\mu}$ and a simple root $\alpha$ such that $\lambda-p \sigma=$ $s_{\alpha} \cdot \bar{\mu}$ and $0 \leq\left\langle\bar{\mu}, \alpha^{\vee}\right\rangle<p-1$. Moreover, $H^{1}(\lambda-p \sigma) \cong H^{0}(\bar{\mu})$.

Since $\operatorname{Hom}_{G}\left(V(\mu), H^{0}(\bar{\mu})\right) \neq 0$ it follows that $\bar{\mu}=\mu$. Thus, the above equation becomes $\lambda-p \sigma=s_{\alpha} \cdot \mu$. Solving for $p \sigma$ yields

$$
p \sigma=\lambda-\mu+\left\langle\mu+\rho, \alpha^{\vee}\right\rangle \alpha .
$$

Taking the inner product with $\alpha^{\vee}$ yields

$$
p\left\langle\sigma, \alpha^{\vee}\right\rangle=\left\langle\lambda-\mu, \alpha^{\vee}\right\rangle+2\left\langle\mu+\rho, \alpha^{\vee}\right\rangle,
$$

which can be written as

$$
p\left\langle\sigma, \alpha^{\vee}\right\rangle=\left\langle\lambda+\rho, \alpha^{\vee}\right\rangle+\left\langle\mu+\rho, \alpha^{\vee}\right\rangle .
$$

Now $\lambda$ and $\mu$ are restricted and $\left\langle\mu+\rho, \alpha^{\vee}\right\rangle\left\langle p\right.$. That forces $\left\langle\sigma, \alpha^{\vee}\right\rangle=1$. Next we assume that $\alpha$ is not the unique long simple root in a root system of type $A_{1}$ or $C_{n}$. Then there exists a positive $\operatorname{root} \beta$ with $\left\langle\alpha, \beta^{\vee}\right\rangle=-1$. We take the inner product with $\beta^{\vee}$ of equation (5.2.1) and obtain

$$
p\left\langle\sigma, \beta^{\vee}\right\rangle=\left\langle\lambda-\mu, \beta^{\vee}\right\rangle-\left\langle\mu+\rho, \alpha^{\vee}\right\rangle .
$$

Adding equation (5.2.4) twice to equation (5.2.2) yields

$$
p+2 p\left\langle\sigma, \beta^{\vee}\right\rangle=\left\langle\lambda-\mu, \alpha^{\vee}\right\rangle+2\left\langle\lambda-\mu, \beta^{\vee}\right\rangle .
$$

The left-hand side is a nonzero multiple of $p$ while the absolute value of the righthand side is less than $p$. We obtain a contradiction. The root system is therefore of type $A_{1}$ or $C_{n}$ and $\alpha$ is the unique long simple root, which we denote by $\alpha_{n}$. Taking the inner product of (5.2.1) with any of the simple roots $\alpha_{1}$ through $\alpha_{n-2}$ yields $p\left\langle\sigma, \alpha_{i}^{\vee}\right\rangle=\left\langle\lambda-\mu, \alpha_{i}^{\vee}\right\rangle$ and thus $\left\langle\sigma, \alpha_{i}^{\vee}\right\rangle=0$. The inner product with $\alpha_{n-1}$ yields

$$
p\left\langle\sigma, \alpha_{n-1}^{\vee}\right\rangle=\left\langle\lambda-\mu, \alpha_{n-1}^{\vee}\right\rangle-2\left\langle\mu+\rho, \alpha_{n}^{\vee}\right\rangle .
$$

Adding (5.2.6) to (5.2.2) yields $p\left\langle\sigma, \alpha_{n-1}^{\vee}\right\rangle+p=\left\langle\lambda-\mu, \alpha_{n-1}^{\vee}\right\rangle+\left\langle\lambda-\mu, \alpha_{n}^{\vee}\right\rangle$. This forces $\left\langle\sigma, \alpha_{n-1}^{\vee}\right\rangle=-1$ and $\sigma=\alpha_{n} / 2$. (Note that in type $A_{1}$ the latter conclusion follows immediately from $\left\langle\sigma, \alpha^{\vee}\right\rangle=1$.) Now equation (5.2.1) can be rewritten as $\lambda=\mu+\left(\frac{p}{2}-\left\langle\mu+\rho, \alpha_{n}^{\vee}\right\rangle\right) \alpha_{n}$ as claimed.

Case 2. There exists a dominant weight $\bar{\mu}$, a unique simple root $\alpha$, and integers $n>0$ and $0<a<p$, such that $\lambda-p \sigma=\bar{\mu}-a p^{n} \alpha$. Moreover, the socle of $H^{1}(\lambda-p \sigma)$ is $L(\bar{\mu})$. 
Since $\operatorname{Hom}_{G}\left(V(\mu), H^{1}(\lambda-p \sigma)\right) \neq 0$ it follows that $\bar{\mu} \uparrow \mu$. We rewrite the above equation as

$$
\bar{\mu}-\lambda=p\left(a p^{n-1} \alpha-\sigma\right) .
$$

Taking the inner product with any simple root $\beta$ results in

$$
-(p-1) \leq\left\langle\bar{\mu}-\lambda, \beta^{\vee}\right\rangle=p\left\langle a p^{n-1} \alpha-\sigma, \beta^{\vee}\right\rangle .
$$

Therefore $a p^{n-1} \alpha-\sigma$ is a dominant weight. Taking the inner product with $\alpha_{0}^{\vee}$ yields

$$
p / 3>\left\langle\mu-\lambda, \alpha_{0}^{\vee}\right\rangle \geq\left\langle\bar{\mu}-\lambda, \alpha_{0}^{\vee}\right\rangle=p\left\langle a p^{n-1} \alpha-\sigma, \alpha_{0}^{\vee}\right\rangle,
$$

which forces $\left\langle a p^{n-1} \alpha-\sigma, \alpha_{0}^{\vee}\right\rangle \leq 0$. Thus $a p^{n-1} \alpha-\sigma$ is the zero weight and $\lambda=\bar{\mu} \uparrow \mu$. The last statement gives the desired contradiction.

The proof of part (b) is identical to that of (a) up to equation (5.2.1) of Case 1. Denote the simple roots by $\alpha_{1}, \alpha_{2}, \ldots, \alpha_{n}$. Then equation (5.2.1) becomes

$$
p \sigma=\alpha+\left\langle\mu+\rho, \alpha_{i}^{\vee}\right\rangle \alpha_{i}
$$

for some simple root $\alpha_{i}$. Therefore the weight $p \sigma$ is in the root lattice. Let us first assume that the root system is not of type $A_{n}$. Then the condition $p \geq h$ implies that $\sigma$ itself is in the root lattice. So there are nonnegative integers $c_{j}$ with $\sigma=$ $\sum_{j=1}^{n} c_{j} \alpha_{j}$. Similarly, one can find nonnegative integers $a_{j}$ with $\alpha=\sum_{j=1}^{n} a_{j} \alpha_{j}$. Since $\alpha$ is not simple, there exists at least one $j \neq i$ with $a_{j} \neq 0$. It follows now from (5.2.10) that $a_{j}=p c_{j} \geq p \geq h$, which is not possible. Now assume that the root system is of type $A_{n}$ and $p>3$. Notice that the statement is empty for type $A_{1}$. Therefore, there exists a simple root $\alpha_{j}$ with $\left\langle\alpha_{i}, \alpha_{j}^{\vee}\right\rangle=-1$. We take the inner product with $\alpha_{j}^{\vee}$ of equation (5.2.10) and obtain the following equation corresponding to (5.2.4):

$$
p\left\langle\sigma, \alpha_{j}^{\vee}\right\rangle=\left\langle\alpha, \alpha_{j}^{\vee}\right\rangle-\left\langle\mu+\rho, \alpha_{i}^{\vee}\right\rangle .
$$

The equivalent to equation (5.2.5) now reads

$$
p+2 p\left\langle\sigma, \alpha_{j}^{\vee}\right\rangle=\left\langle\alpha, \alpha_{i}^{\vee}\right\rangle+2\left\langle\alpha, \alpha_{j}^{\vee}\right\rangle .
$$

For type $A_{n}$ the absolute value of an inner product of a positive nonsimple root with a simple root is at most 1 . The absolute value of the right-hand side of (5.2.12) is less than or equal to 3 while the left-hand side is a nonzero multiple of $p$. Now $p>3$ produces the desired contradiction.

For the proof of Case 2, we can simply copy the argument from Case 2 of part (a). Notice that equation (5.2.9) can be replaced by the stronger equation

$$
0 \geq\left\langle-\alpha, \alpha_{0}^{\vee}\right\rangle=\left\langle\mu-\lambda, \alpha_{0}^{\vee}\right\rangle \geq\left\langle\bar{\mu}-\lambda, \alpha_{0}^{\vee}\right\rangle=p\left\langle a p^{n-1} \alpha-\sigma, \alpha_{0}^{\vee}\right\rangle .
$$

5.3. The following theorem shows that for groups other than types $A_{1}$ or $C_{n}$ and for pairs of simple modules whose highest weights are restricted and "close together" the $G$ - and $G_{1}$-extensions coincide.

Theorem (A). Let $G$ be a simple algebraic group, $p>3, \lambda, \mu \in X_{1}(T)$. If $\left\langle\lambda-\mu, \alpha^{\vee}\right\rangle<p / 3$ for all roots $\alpha$, then one of the following holds:

(a) $\operatorname{Ext}_{G_{1}}^{1}(L(\lambda), L(\mu)) \cong \operatorname{Ext}_{G}^{1}(L(\lambda), L(\mu))$.

(b) The underlying root system is of type $A_{1}$ or $C_{n}$ and $\mu=\lambda+\left(\frac{p}{2}-\left\langle\lambda+\rho, \alpha_{n}^{\vee}\right\rangle\right) \alpha_{n}$, where $\alpha_{n}$ is the unique long (last) simple root. 
Proof. The case $\lambda=\mu$ was done in [And1, Thm. 4.5]. We may assume that $\lambda \neq \mu$. It follows immediately from Proposition 5.2 that $\operatorname{Ext}_{G_{1}}^{1}\left(V(\lambda), H^{0}(\mu)\right)=0$ or that the underlying root system is of type $A_{1}$ or $C_{n}$ and $\mu=\lambda+\left(\frac{p}{2}-\left\langle\lambda+\rho, \alpha_{n}^{\vee}\right\rangle\right) \alpha_{n}$, where $\alpha_{n}$ is the unique long (last) simple root. In the first case, we wish to apply Lemma 5.1. For any root $\alpha$ and dominant weight $\nu \neq 0$, one has the following sequence of inequalities:

$$
\left\langle\lambda, \alpha_{0}^{\vee}\right\rangle \leq\left\langle\mu, \alpha_{0}^{\vee}\right\rangle+p / 3<\left\langle\mu, \alpha_{0}^{\vee}\right\rangle+p \leq\left\langle\mu+p \nu, \alpha_{0}^{\vee}\right\rangle .
$$

Thus $\mu+p \nu \nless \lambda$ and similarly $\lambda+p \nu \nless \mu$. Hence, the claim follows from Lemma 5.1 .

Using the notation of Section 2.1, we can now prove a generalization of Corollary 3.3 in [BNP2] and give sufficient conditions to insure that $\operatorname{Ext}_{G\left(\mathbb{F}_{q}\right)}^{1}(L(\lambda), L(\mu)) \cong$ $\operatorname{Ext}_{G}^{1}(L(\lambda), L(\mu))$ and, moreover, that these extensions can also be identified with $\operatorname{Ext}_{G_{r}}^{1}(L(\lambda), L(\mu))$.

Theorem (B). Let $G$ be a simple simply connected algebraic group with $p \geq$ $3(h-1), r \geq 2$, and $\lambda, \mu \in X_{r}(T)$. If $\left\langle\lambda_{r-1}-\mu_{r-1}, \alpha^{\vee}\right\rangle<p / 3$ for all roots $\alpha$ and either the underlying root system is not of type $A_{1}$ or $C_{n}$ or $\mu_{r-1} \neq \lambda_{r-1}+$ $\left(\frac{p}{2}-\left\langle\lambda_{r-1}+\rho, \alpha_{n}^{\vee}\right\rangle\right) \alpha_{n}$, where $\alpha_{n}$ is the unique long (last) simple root, then

$$
\operatorname{Ext}_{G\left(\mathbb{F}_{q}\right)}^{1}(L(\lambda), L(\mu)) \cong \operatorname{Ext}_{G}^{1}(L(\lambda), L(\mu)) \cong \operatorname{Ext}_{G_{r}}^{1}(L(\lambda), L(\mu)) .
$$

Proof. For $G\left(\mathbb{F}_{q}\right)$, by Theorem 2.1(b), we have

$$
\operatorname{Ext}_{G\left(\mathbb{F}_{q}\right)}^{1}(L(\lambda), L(\mu)) \cong \operatorname{Ext}_{G}^{1}(L(\lambda), L(\mu)) \oplus R
$$

where

$$
\begin{aligned}
R= & \bigoplus_{\nu \in \Gamma-\{0\}} \operatorname{Hom}_{G}\left(L(\nu), \operatorname{Ext}_{G_{1}}^{1}\left(L\left(\lambda_{r-1}\right), L\left(\mu_{r-1}\right)\right)^{(-1)}\right) \\
& \otimes \operatorname{Hom}_{G}(L(\dot{\lambda}), L(\dot{\mu}) \otimes L(\nu)) .
\end{aligned}
$$

So it suffices to show that the remainder term $R$ is zero. By Theorem 5.3(A), one obtains that $\operatorname{Ext}_{G_{1}}^{1}\left(L\left(\lambda_{r-1}\right), L\left(\mu_{r-1}\right)\right) \cong \operatorname{Ext}_{G}^{1}\left(L\left(\lambda_{r-1}\right), L\left(\mu_{r-1}\right)\right)$. Hence for all $\nu \neq 0$ the term $\operatorname{Hom}_{G}\left(L(\nu), \operatorname{Ext}_{G_{1}}^{1}\left(L\left(\lambda_{r-1}\right), L\left(\mu_{r-1}\right)\right)^{(-1)}\right)$ equals zero and $R=0$. The isomorphism for $G_{r}$ follows immediately from Corollary 2.7(A) and Theorem $5.3(\mathrm{~A})$.

5.4. We now present some other consequences of Theorem 5.3(A).

Corollary (A). Let $G$ be a simple algebraic group. If $\lambda, \mu \in X_{1}(T)$ are p-regular weights contained in the same alcove, then one of the following holds:

(i) $\operatorname{Ext}_{G_{1}}^{1}(L(\lambda), L(\mu))=0$.

(ii) The underlying root system is of type $A_{1}$ or $C_{n}$ and $\mu=\lambda+\left(\frac{p}{2}-\left\langle\lambda+\rho, \alpha_{n}^{\vee}\right\rangle\right) \alpha_{n}$, where $\alpha_{n}$ is the unique long (last) simple root.

Proof. As in Theorem 5.3(A), we may assume that $\lambda \neq \mu$. In order to have two distinct $p$-regular weights located in the same alcove, one needs $p>h$. Suppose $\lambda$ and $\mu$ are two such $p$-regular weights located in the alcove $A$ and $\operatorname{Ext}_{G_{1}}^{1}(L(\lambda), L(\mu)) \neq 0$. Then $\lambda$ and $\mu$ must be $G_{1}$-linked, i.e., there is some $w \in W$ and $\gamma \in X(T)$ such that $\mu=w \cdot \lambda+p \gamma$. Let $\lambda_{C}$ and $\mu_{C}$ be the representatives in the lowest dominant alcove $C$ of the $\lambda$ and $\mu$ linkage classes. Then $\lambda_{C}$ and $\mu_{C}$ are also $G_{1}$-linked. As above, suppose $\mu_{C}=w^{\prime} \cdot \lambda_{C}+p \gamma^{\prime}$. We set $X_{\mathbb{Q}}=X(T) \otimes_{\mathbb{Z}} \mathbb{Q}$ and denote by $A_{\mathbb{Q}}$ 
and $C_{\mathbb{Q}}$ the subsets of $X_{\mathbb{Q}}$ that correspond to $A$ and $C$, respectively. For $x \in C_{\mathbb{Q}}$, set $\phi(x)=w^{\prime} \cdot x+p \gamma^{\prime}$. Then $\phi\left(C_{\mathbb{Q}}\right)=C_{\mathbb{Q}}$. For each $k>0$, we define the following subsets of $C_{\mathbb{Q}}$ :

$$
I_{k}=\left\{x \in C_{\mathbb{Q}} \mid k \leq\left\langle x+\rho, \alpha^{\vee}\right\rangle \leq p-k \text { for all } \alpha \in \Phi^{+}\right\} .
$$

The sets $I_{k}$ are the subsets of all points in $C_{\mathbb{Q}}$ that have distance at least $k$ from any alcove wall. The isometry $\phi$ leaves the sets $I_{k}$ invariant. Moreover, it follows from $\left\langle\frac{p-h}{h} \rho+\rho, \alpha_{0}^{\vee}\right\rangle=\frac{p}{h}(h-1)=p-\frac{p}{h}$, that the set $I_{p / h}$ contains just one point, namely $P=\frac{p-h}{h} \rho$. Thus $P$ is a fixed point of $\phi$.

Consider now the affine Weyl group $W_{1}$, consisting of the ordinary Weyl group and translations by weights. The action of $W_{1}$ subdivides the alcove $C_{\mathbb{Q}}$ into "mini"alcoves. Set $D_{\mathbb{Q}}=\left\{x \in C_{\mathbb{Q}} \mid 0<\left\langle x+\rho, \alpha^{\vee}\right\rangle<1\right.$ for all $\left.\alpha \in \Phi^{+}\right\}$. The set $D_{\mathbb{Q}}$ is the lowest mini-alcove inside $C_{\mathbb{Q}}$. For any two points $M$ and $N$ in the closure of $D_{\mathbb{Q}}$ and any root $\alpha$, we have $\left|\left\langle M-N, \alpha^{\vee}\right\rangle\right| \leq \max \left\{\left\langle M+\rho, \alpha_{0}^{\vee}\right\rangle,\left\langle N+\rho, \alpha_{0}^{\vee}\right\rangle\right\} \leq 1$. Since the elements of $W_{1}$ permute these mini-alcoves within $X_{\mathbb{Q}}$, it follows for any two points $M, N$ in the closure of the same mini-alcove and all roots $\alpha$ that $\left|\left\langle M-N, \alpha^{\vee}\right\rangle\right| \leq 1$. Moreover, the closure of $D_{\mathbb{Q}}$ as well as the closure of every mini-alcove contain some weights. For any positive root $\alpha$, there exists an integer $m<h$ such that $\left\langle\frac{p-h}{h} \rho+\rho, \alpha^{\vee}\right\rangle=\frac{p m}{h}$. Clearly $h$ divides neither $m$ nor $p$. Therefore, $P$ lies in the interior of some mini-alcove, denoted by $E_{\mathbb{Q}}$. The map $\phi$ permutes the mini-alcoves within $C_{\mathbb{Q}}$. Therefore, $\phi$ maps $E_{\mathbb{Q}}$ onto itself. Pick a weight $\lambda_{C}^{\prime}$ in the closure of $E_{\mathbb{Q}}$ and denote $\phi\left(\lambda_{C}^{\prime}\right)$ by $\mu_{C}^{\prime}$. Now $\left|\left\langle\mu_{C}^{\prime}-\lambda_{C}^{\prime}, \alpha^{\vee}\right\rangle\right| \leq 1$ for all roots $\alpha$. The point $P$ has distance $\frac{p}{h}>1$ from the walls of $C_{\mathbb{Q}}$. Hence, the closure of the mini-alcove $E_{\mathbb{Q}}$ lies in the interior of $C_{\mathbb{Q}}$ and the weights $\lambda_{C}^{\prime}$ and $\mu_{C}^{\prime}$ are $p$-regular.

We denote by $\lambda^{\prime}$ and by $\mu^{\prime}$ the representatives of the $\lambda_{C}^{\prime}$ and $\mu_{C}^{\prime}$ linkage classes in the alcove $A$. Then $\lambda^{\prime}$ and $\mu^{\prime}$ are $p$-regular weights with $\mu^{\prime}=w \cdot \lambda^{\prime}+p \gamma$ and $\left|\left\langle\mu^{\prime}-\lambda^{\prime}, \alpha^{\vee}\right\rangle\right| \leq 1$ for all roots $\alpha$.

We assume that $\operatorname{Ext}_{G_{1}}^{1}(L(\lambda), L(\mu)) \neq 0$. Then there exists a dominant weight $\nu$ with $\operatorname{Hom}_{G}\left(L(\nu)^{(1)}, \operatorname{Ext}_{G_{1}}^{1}(L(\lambda), L(\mu))\right) \neq 0$. The weights $\lambda, \lambda^{\prime}, \mu$ and $\mu^{\prime}$ are $p$ restricted and $\lambda^{\prime} \neq \mu^{\prime}$. Therefore, $\operatorname{Hom}_{G_{1}}(L(\lambda), L(\mu))=\operatorname{Hom}_{G_{1}}\left(L\left(\lambda^{\prime}\right), L\left(\mu^{\prime}\right)\right)=0$. We may apply the Lyndon-Hochschild-Serre exact sequence for $G_{1} \subset G$ as in the proof of Proposition 4.3 and obtain

$$
\begin{aligned}
0 \neq \operatorname{Hom}_{G}\left(L(\nu)^{(1)}, \operatorname{Ext}_{G_{1}}^{1}(L(\lambda), L(\mu))\right) & \cong \operatorname{Ext}_{G}^{1}\left(L(\lambda) \otimes L(\nu)^{(1)}, L(\mu)\right) \\
& \cong \operatorname{Ext}_{G}^{1}\left(L(\lambda), L\left(\mu-p w_{0} \nu\right)\right) .
\end{aligned}
$$

Recall that $\mu=w \cdot \lambda+p \gamma$, which implies that $\mu-p w_{0} \nu=w \cdot \lambda+p\left(\gamma-w_{0} \nu\right)$. Similarly, $\mu^{\prime}-p w_{0} \nu=w \cdot \lambda^{\prime}+p\left(\gamma-w_{0} \nu\right)$. The weight $\gamma-w_{0} \nu$ is in the root lattice because the weights $\lambda$ and $\mu-p w_{0} \nu$ are linked. Hence, the weights $\lambda^{\prime}$ and $\mu^{\prime}-p w_{0} \nu$ are also linked. We use the adjointness of the translation functors $T_{\lambda}^{\lambda^{\prime}}$ and $T_{\lambda^{\prime}}^{\lambda}$ to obtain

$$
\begin{aligned}
0 & \neq \operatorname{Ext}_{G}^{1}\left(L(\lambda), L\left(\mu-p w_{0} \nu\right)\right) \\
\cong & \operatorname{Ext}_{G}^{1}\left(T_{\lambda^{\prime}}^{\lambda} L\left(\lambda^{\prime}\right), L\left(\mu-p w_{0} \nu\right)\right) \\
& \cong \operatorname{Ext}_{G}^{1}\left(L\left(\lambda^{\prime}\right), T_{\lambda}^{\lambda^{\prime}} L\left(\mu-p w_{0} \nu\right)\right) \\
& \cong \operatorname{Ext}_{G}^{1}\left(L\left(\lambda^{\prime}\right), L\left(\mu^{\prime}-p w_{0} \nu\right)\right) \\
& \cong \operatorname{Ext}_{G}^{1}\left(L\left(\lambda^{\prime}\right) \otimes L(\nu)^{(1)}, L\left(\mu^{\prime}\right)\right) \\
& \cong \operatorname{Hom}_{G}\left(L(\nu)^{(1)}, \operatorname{Ext}_{G_{1}}^{1}\left(L\left(\lambda^{\prime}\right), L\left(\mu^{\prime}\right)\right)\right)
\end{aligned}
$$


Now $\operatorname{Ext}_{G_{1}}^{1}\left(L\left(\lambda^{\prime}\right), L\left(\mu^{\prime}\right)\right) \neq 0$. For all roots $\alpha$, we have $\left|\left\langle\mu^{\prime}-\lambda^{\prime}, \alpha^{\vee}\right\rangle\right| \leq 1<\frac{p}{3}$. We apply Theorem 5.3(A) and conclude that the underlying root system is of type $A_{1}$ or $C_{n}$.

Moreover, for type $A_{1}$ or $C_{n}$, it follows that $\mu^{\prime}=\lambda^{\prime}+\left(\frac{p}{2}-\left\langle\lambda^{\prime}+\rho, \alpha_{n}^{\vee}\right\rangle\right) \alpha_{n}=$ $s_{\alpha_{n}} \cdot \lambda^{\prime}+p\left(\alpha_{n} / 2\right)$. Thus $\mu=s_{\alpha_{n}} \cdot \lambda+p\left(\alpha_{n} / 2\right)=\lambda+\left(\frac{p}{2}-\left\langle\lambda+\rho, \alpha_{n}^{\vee}\right\rangle\right) \alpha_{n}$ as claimed. Note that for type $A_{1}$, the condition $p>h$ allows the case $p=3$ so that Theorem 5.3(A) does not apply. However, for $p=3$, one readily checks that the above relation between $\mu$ and $\lambda$ must hold.

Corollary (B). Let $G$ be a simple simply connected algebraic group. When $r=1$, assume that the underlying root system is not of type $A_{1}$.

(i) If $p>2$, then $\operatorname{Ext}_{G_{r}}^{1}(L(\lambda), L(\mu))=0$ for all $\lambda, \mu \in \bar{C}_{\mathbb{Z}}$.

(ii) If $p \geq 3(h-1)$, then $\operatorname{Ext}_{G\left(\mathbb{F}_{q}\right)}^{1}(L(\lambda), L(\mu))=0$ for all $\lambda, \mu \in C_{\mathbb{Z}}$.

Proof. (i) The case $\lambda=\mu$ was done in [And1, Thm. 4.5]. We may assume that $\lambda \neq \mu$. Let $\lambda, \mu \in \bar{C}_{\mathbb{Z}}$. It follows from [And1, Thm. 5.3] that $\operatorname{Ext}_{G_{r}}^{1}(L(\lambda), L(\mu))=0$ implies $\operatorname{Ext}_{G_{1}}^{1}(L(\lambda), L(\mu))=0$. Assume that $\operatorname{Ext}_{G_{1}}^{1}(L(\lambda), L(\mu)) \neq 0$. Then the weights $\lambda$ and $\mu$ are $G_{1}$-linked. This implies that either both weights are $p$-singular or both weights are $p$-regular. If they are both $p$-singular they have to be contained in the upper wall of the bottom fundamental alcove. Clearly they are not $G$-linked. We will show that no two weights in the upper wall can be $G_{1}$-linked. Let $\widetilde{W}_{p}$ denote the affine Weyl group generated by $W$ and the translations by elements of $p X(T)$. Any nonzero element of the quotient group $\widetilde{W}_{p} / W_{p}$ defines an action that fixes the bottom alcove and permutes its vertices. In particular, it maps the vertex $-\rho$ into the upper wall. In return, one of the vertices in the upper wall gets mapped back to $-\rho$. The upper wall is not invariant under the action. Therefore two weights that are $G_{1}$-linked, i.e., one is the image of the other under the action of some element in $\widetilde{W}_{p}$, cannot both be in the upper wall.

If the weights are $p$-regular, it follows from Corollary 5.4(A) that the underlying root system of $G$ is of type $A_{1}$ or $C_{n}$ and that $\mu=\lambda+\left(\frac{p}{2}-\left\langle\lambda+\rho, \alpha_{n}^{\vee}\right\rangle\right) \alpha_{n}$, where $\alpha_{n}$ is the unique long (last) simple root. For type $C_{n}, n \geq 2$, one of the weights, say $\mu$, satisfies $\left\langle\mu+\rho, \alpha_{n}^{\vee}\right\rangle>p / 2$. But this implies that $\left\langle\mu+\rho, \alpha_{0}^{\vee}\right\rangle>p$ and so $\mu$ is not contained in the closure of the bottom alcove, which contradicts the assumption. For type $A_{1}$ and $r \geq 2$, we have $\operatorname{Ext}_{G_{r}}^{1}(L(\lambda), L(\mu))=0$; see [AJL].

(ii) For $r>1$, the statement follows from Theorem 4.8(A) except in the case that $p=3$, the root system is of Type $A_{1}$, and $\lambda=\mu=2$. However, in that case, there are no self-extensions by [AJL BNP2]. For $r=1$, we apply Theorem 2.1(c) and obtain

$$
\operatorname{Ext}_{G\left(\mathbb{F}_{p}\right)}^{1}(L(\lambda), L(\mu)) \cong \operatorname{Ext}_{G}^{1}(L(\lambda), L(\mu)) \oplus R
$$

where

$$
R=\bigoplus_{\nu \in \Gamma-\{0\}} \operatorname{Hom}_{G}\left(L(\nu)^{(1)}, \operatorname{Ext}_{G_{1}}^{1}(L(\lambda), L(\mu) \otimes L(\nu))\right) .
$$

Clearly $\operatorname{Ext}_{G}^{1}(L(\lambda), L(\mu))=0$. Using [And2, Lemma 2.3], we conclude that any $\nu$ that contributes to the summation of $R$ has to satisfy $(p-1)\left\langle\nu, \alpha_{0}^{\vee}\right\rangle \leq\left\langle-w_{0} \lambda+\mu+\right.$ $\left.\alpha, \alpha_{0}^{\vee}\right\rangle$ for some simple root $\alpha$. It follows that $(p-1)\left\langle\nu, \alpha_{0}^{\vee}\right\rangle<2(p-(h-1))+2$, which is less than $2(p-1)$ unless $\Phi$ is of type $A_{1}$. Therefore $\nu$ is a minuscule weight. This implies that all composition factors $L(\gamma)$ of $L(\mu) \otimes L(\nu)$ are inside 
$\bar{C}_{\mathbb{Z}}$. We apply part (a) and obtain for all $\gamma$ that $\operatorname{Ext}_{G_{1}}^{1}(L(\lambda), L(\gamma))=0$. Hence $\operatorname{Ext}_{G_{1}}^{1}(L(\lambda), L(\mu) \otimes L(\nu))=0$ and also $R=0$.

In CPS2, Thm. 5.3] it is shown that the Lusztig conjecture is equivalent to the assertion that $\operatorname{Ext}_{G}^{1}(L(\lambda), L(\mu)) \neq 0$ for certain dominant weights $\lambda$ and $\mu$ that are mirror images of each other in adjacent alcoves. The following corollary shows that for such $p$-restricted pairs the $G$-extensions and $G_{1}$-extensions coincide.

Corollary (C). Let $G$ be a simple algebraic group, $p \geq h\left(p>3\right.$ for type $\left.A_{2}\right)$, $\lambda, \mu$-regular weights in $X_{1}(T)$. If $\lambda$ and $\mu$ are mirror images of one another in adjacent alcoves, then

$$
\operatorname{Ext}_{G_{1}}^{1}(L(\lambda), L(\mu)) \cong \operatorname{Ext}_{G}^{1}(L(\lambda), L(\mu))
$$

Proof. Suppose that $\lambda$ and $\mu$ are reflections of one another with respect to a hyperplane $H_{(\alpha, m p)}=\left\{x \in X(T)_{+} \otimes_{\mathbb{Z}} \mathbb{R} \mid\left\langle x+\rho, \alpha^{\vee}\right\rangle=m p\right\}$, where $\alpha$ is some positive root. Notice that $\alpha$ is not a simple root because both $\lambda$ and $\mu$ are assumed to be $p$-restricted. We can find weights $\lambda^{\prime}$ and $\mu^{\prime}$ that are mirror images of one another in the same adjacent alcoves such that $\lambda^{\prime}=\mu^{\prime}+\alpha$. We apply Proposition 5.2(b) and conclude that $\operatorname{Ext}_{G_{1}}^{1}\left(V\left(\lambda^{\prime}\right), H^{0}\left(\mu^{\prime}\right)\right)=0$. (In type $A_{1}$, no such $\lambda$ and $\mu$ exist.) Now it follows from Lemma 5.1 that

$$
\operatorname{Ext}_{G_{1}}^{1}\left(L\left(\lambda^{\prime}\right), L\left(\mu^{\prime}\right)\right) \cong \operatorname{Ext}_{G}^{1}\left(L\left(\lambda^{\prime}\right), L\left(\mu^{\prime}\right)\right) .
$$

As in the proof of Corollary 5.4(A), one applies the adjointness of the translation functors $T_{\lambda}^{\lambda^{\prime}}$ and $T_{\lambda^{\prime}}^{\lambda}$ to obtain

$$
\begin{aligned}
\operatorname{Ext}_{G_{1}}^{1}(L(\lambda), L(\mu)) \cong \operatorname{Ext}_{G_{1}}^{1}\left(L\left(\lambda^{\prime}\right), L\left(\mu^{\prime}\right)\right) & \cong \operatorname{Ext}_{G}^{1}\left(L\left(\lambda^{\prime}\right), L\left(\mu^{\prime}\right)\right) \\
& \cong \operatorname{Ext}_{G}^{1}(L(\lambda), L(\mu)) .
\end{aligned}
$$

Example. Let $G=S L_{3}$ and $p=3=h$, and let $\omega_{1}, \omega_{2}$ be the fundamental weights. We note that the conclusion of Corollary 5.4(C) fails to hold here. Specifically, $\rho=\omega_{1}+\omega_{2}$ is the refection of the zero weight across the upper wall of the bottom dominant alcove. Yet

$$
\mathrm{H}^{1}(G, L(\rho)) \varsubsetneqq \mathrm{H}^{1}\left(G_{1}, L(\rho)\right)
$$

since $L(\rho)$ admits extensions (over $G)$ by $k, L\left(\omega_{1}\right)^{(1)}$, and $L\left(\omega_{2}\right)^{(1)}$, and the latter modules are trivial over $G_{1}$.

5.5. In Sections 5.6 and 5.7, we present some other conditions under which $G\left(\mathbb{F}_{q}\right)$ and/or $G_{r}$-extensions may be identified with $G$-extensions. We begin with some preliminary results.

Lemma. Let $G$ be a simple algebraic group, $\lambda, \mu \in X_{1}(T)$, and $\nu_{1}, \nu_{2} \in X(T)_{+}$. If $\lambda+p \nu_{1} \nless \mu+\nu_{2}$, then there exists an embedding

$$
\operatorname{Ext}_{G}^{1}\left(L\left(\lambda+p \nu_{1}\right), L(\mu) \otimes L\left(\nu_{2}\right)\right) \hookrightarrow \operatorname{Ext}_{G}^{1}\left(L\left(\lambda+p \nu_{1}\right), H^{0}(\mu) \otimes L\left(\nu_{2}\right)\right) .
$$

Proof. There exists a short exact sequence of the form

$$
0 \rightarrow L(\mu) \otimes L\left(\nu_{2}\right) \rightarrow H^{0}(\mu) \otimes L\left(\nu_{2}\right) \rightarrow M \rightarrow 0
$$

This induces a long exact sequence

$$
\begin{aligned}
\cdots & \rightarrow \operatorname{Hom}_{G}\left(L\left(\lambda+p \nu_{1}\right), M\right) \rightarrow \operatorname{Ext}_{G}^{1}\left(L\left(\lambda+p \nu_{1}\right), L(\mu) \otimes L\left(\nu_{2}\right)\right) \\
& \rightarrow \operatorname{Ext}_{G}^{1}\left(L\left(\lambda+p \nu_{1}\right), H^{0}(\mu) \otimes L\left(\nu_{2}\right)\right) \rightarrow \cdots .
\end{aligned}
$$


The composition factors of $M$ have highest weights $\sigma$ satisfying $\sigma<\mu+\nu_{2}$. By assumption, we have $\lambda+p \nu_{1} \nless \mu+\nu_{2}$. Hence, $\operatorname{Hom}_{G}\left(L\left(\lambda+p \nu_{1}\right), M\right)=0$, which gives the claimed embedding.

Proposition. Let $G$ be a simple algebraic group with $p \geq h, \lambda, \mu \in X_{1}(T)$, and $\nu_{1}, \nu_{2} \in \Gamma$ with $\nu_{1} \neq 0$. Suppose that for any weights $\gamma$ of $L\left(-w_{0} \lambda\right)$ and $\eta$ of $L\left(\nu_{2}\right)$ the weight $\mu+\gamma+\eta-p \nu_{1}$ is not equal to either of the following:

(a) $-\alpha$,

(b) $-p\left\langle\nu_{1}+\rho, \alpha^{\vee}\right\rangle \alpha$,

for a simple root $\alpha$. Then $\operatorname{Ext}_{G}^{1}\left(L\left(\lambda+p \nu_{1}\right), H^{0}(\mu) \otimes L\left(\nu_{2}\right)\right)=0$.

Proof. The module $H^{0}(\mu) \otimes L\left(\nu_{2}\right) \cong H^{0}(\mu) \otimes H^{0}\left(\nu_{2}\right)$ has a filtration with sections of the form $H^{0}(\mu+\eta)$ where $\eta$ is a weight of $L\left(\nu_{2}\right)$. To show the claim, it suffices to prove that $\operatorname{Ext}_{G}^{1}\left(L\left(\lambda+p \nu_{1}\right), H^{0}(\mu+\eta)\right)=0$ for all $\mu+\eta \in X(T)_{+}$such that $\eta$ is a weight of $L\left(\nu_{2}\right)$. Observe that

$$
\begin{aligned}
\operatorname{Ext}_{G}^{1}\left(L\left(\lambda+p \nu_{1}\right), H^{0}(\mu+\eta)\right) & \cong \operatorname{Ext}_{B}^{1}\left(L\left(\lambda+p \nu_{1}\right), \mu+\eta\right) \\
& \cong \operatorname{Ext}_{B}^{1}\left(L\left(\nu_{1}\right)^{(1)},(\mu+\eta) \otimes L\left(-w_{0} \lambda\right)\right) .
\end{aligned}
$$

We are now reduced to showing that $\operatorname{Ext}_{B}^{1}\left(L\left(\nu_{1}\right)^{(1)}, \mu+\eta+\gamma\right)=0$ for $\eta$ a weight of $L\left(\nu_{2}\right)$ and $\gamma$ a weight of $L\left(-w_{0} \lambda\right)$. There are two cases to consider: $\mu+\gamma+\eta \in$ $X(T)_{+}$and $\mu+\gamma+\eta \notin X(T)_{+}$.

Case 1. $\mu+\gamma+\eta \in X(T)_{+}$.

Then

$$
\operatorname{Ext}_{B}^{1}\left(L\left(\nu_{1}\right)^{(1)}, \mu+\gamma+\eta\right) \cong \operatorname{Ext}_{G}^{1}\left(L\left(\nu_{1}\right)^{(1)}, H^{0}(\mu+\gamma+\eta)\right)
$$

and there exists a spectral sequence

$$
\begin{aligned}
E_{2}^{i, j} & =\operatorname{Ext}_{G / G_{1}}^{i}\left(L\left(\nu_{1}\right)^{(1)}, \operatorname{Ext}_{G_{1}}^{j}\left(k, H^{0}(\mu+\gamma+\eta)\right)\right) \\
& \Rightarrow \operatorname{Ext}_{G}^{j}\left(L\left(\nu_{1}\right)^{(1)}, H^{0}(\mu+\gamma+\eta)\right) .
\end{aligned}
$$

Observe that $\operatorname{Hom}_{G_{1}}\left(k, H^{0}(\mu+\gamma+\eta)\right) \cong \operatorname{ind}_{B}^{G} \operatorname{Hom}_{B_{1}}(k, \mu+\gamma+\eta) \cong \operatorname{ind}_{B}^{G} p \sigma$ for some $\sigma \in X(T)$. Therefore, for $i \geq 0$,

$$
\begin{aligned}
E_{2}^{i, 0} & =\operatorname{Ext}_{G / G_{1}}^{i}\left(L\left(\nu_{1}\right)^{(1)}, \operatorname{Hom}_{G_{1}}\left(k, H^{0}(\mu+\gamma+\eta)\right)\right) \\
& \cong \operatorname{Ext}_{G / G_{1}}^{i}\left(V\left(\nu_{1}\right)^{(1)}, \operatorname{ind}_{B}^{G} p \sigma\right) \\
& =0 .
\end{aligned}
$$

Thus, $\operatorname{Ext}_{G}^{1}\left(L\left(\nu_{1}\right)^{(1)}, H^{0}(\mu+\gamma+\eta)\right) \cong \operatorname{Hom}_{G}\left(L\left(\nu_{1}\right)^{(1)}, \operatorname{Ext}_{G_{1}}^{1}\left(k, H^{0}(\mu+\gamma+\eta)\right)\right)$. By Proposition 4.3, if this Hom group is nonzero, we must have $\mu+\gamma+\eta=p \nu_{1}-\alpha$ for some simple root $\alpha$. But that possibility has been excluded.

Case 2. $\mu+\gamma+\eta \notin X(T)_{+}$.

If $\operatorname{Ext}_{B}^{1}\left(L\left(\nu_{1}\right)^{(1)}, \mu+\gamma+\eta\right) \neq 0$, then, by [Jan1] II.5.19(4)], $\mu+\gamma+\eta=p \nu_{1}-c p^{n} \alpha$ for a simple root $\alpha$ and integers $n \geq 0$ and $c<p$. Specifically, $c$ is defined as the unique integer with $(c-1) p^{n}\left\langle\left\langle p \nu_{1}+\rho, \alpha^{\vee}\right\rangle \leq c p^{n}\right.$ (see [Jan1, II.5.17]). Since $\nu_{1}$ is dominant, $c>0$. Observe also that $\left|\left\langle\mu+\gamma+\eta, \alpha^{\vee}\right\rangle\right| \leq 3(h-1) \leq 3(p-1)$ while $\left|\left\langle p \nu_{1}-c p^{n} \alpha, \alpha^{\vee}\right\rangle\right| \geq 2 c p^{n}-p(h-1) \geq 2 c p^{n}-\left(p^{2}-p\right) \geq 2 p^{n}-p^{2}+p$ and hence $n \leq 1$.

Suppose $n=0$. Then $c$ satisfies $c-1<\left\langle p \nu_{1}+\rho, \alpha^{\vee}\right\rangle \leq c$. Hence $c=\left\langle p \nu_{1}+\right.$ $\left.\rho, \alpha^{\vee}\right\rangle=p\left\langle\nu_{1}, \alpha^{\vee}\right\rangle+1$. However, $c<p$ and so we must have $\left\langle\nu_{1}, \alpha^{\vee}\right\rangle=0$ and 
$c=1$. That leaves $\mu+\gamma+\eta=p \nu_{1}-\alpha$, which has been excluded. On the other hand, suppose that $n=1$. Then $c$ satisfies $(c-1) p\left\langle\left\langle p \nu_{1}+\rho, \alpha^{\vee}\right\rangle \leq c p\right.$. Hence $c=\left\langle\nu_{1}, \alpha^{\vee}\right\rangle+1=\left\langle\nu_{1}+\rho, \alpha^{\vee}\right\rangle$ and $\mu+\gamma+\eta=p \nu_{1}-p\left\langle\nu_{1}+\rho, \alpha^{\vee}\right\rangle \alpha$. This case was also excluded.

5.6. For $r \geq 2$, we can now give sufficient conditions to insure that $\operatorname{Ext}_{G\left(\mathbb{F}_{q}\right)}^{1}(L(\lambda), L(\mu))$ and $\operatorname{Ext}_{G_{r}}^{1}(L(\lambda), L(\mu))$ are isomorphic to $\operatorname{Ext}_{G}^{1}(L(\lambda), L(\mu))$ (and hence to each other).

Theorem. Let $G$ be a simple simply connected algebraic group with $p \geq 3(h-1)$, $r \geq 2$, and $\lambda, \mu \in X_{r}(T)$. Suppose that the following conditions hold for any $\nu \in \Gamma-\{0\}:$

(a) $\lambda_{r-1}+p \nu \nless \mu_{r-1}$.

(b) For any weight $\gamma$ of $L\left(-w_{0} \lambda_{r-1}\right), \mu_{r-1}+\gamma-p \nu$ is not equal to either of the following:

(i) $-\alpha$,

(ii) $-p\left\langle\nu+\rho, \alpha^{\vee}\right\rangle \alpha$,

for a simple root $\alpha$. Then

$$
\operatorname{Ext}_{G\left(\mathbb{F}_{q}\right)}^{1}(L(\lambda), L(\mu)) \cong \operatorname{Ext}_{G}^{1}(L(\lambda), L(\mu)) \cong \operatorname{Ext}_{G_{r}}^{1}(L(\lambda), L(\mu)) .
$$

Proof. Consider first the case of $G\left(\mathbb{F}_{q}\right)$. By Theorem 2.1(b), we have

$$
\operatorname{Ext}_{G\left(\mathbb{F}_{q}\right)}^{1}(L(\lambda), L(\mu)) \cong \operatorname{Ext}_{G}^{1}(L(\lambda), L(\mu)) \oplus R
$$

where

$$
R=\bigoplus_{\nu \in \Gamma-\{0\}} \operatorname{Ext}_{G}^{1}\left(L\left(\lambda_{r-1}\right) \otimes L(\nu)^{(1)}, L\left(\mu_{r-1}\right)\right) \otimes \operatorname{Hom}_{G}(L(\dot{\lambda}), L(\dot{\mu}) \otimes L(\nu)) .
$$

We show that $\operatorname{Ext}_{G}^{1}\left(L\left(\lambda_{r-1}\right) \otimes L(\nu)^{(1)}, L\left(\mu_{r-1}\right)\right)=0$ for all $\nu \in \Gamma-\{0\}$ and hence that the remainder term $R$ is zero. By condition (i), we may apply Lemma 5.5 (with $\nu_{1}=\nu$ and $\nu_{2}=0$ ) and conclude that there is an embedding

$$
\operatorname{Ext}_{G}^{1}\left(L\left(\lambda_{r-1}\right) \otimes L(\nu)^{(1)}, L(\mu)\right) \hookrightarrow \operatorname{Ext}_{G}^{1}\left(L\left(\lambda_{r-1}\right) \otimes L(\nu)^{(1)}, H^{0}(\mu)\right)
$$

for all $\nu \in \Gamma-\{0\}$. Further, condition (ii) implies that the hypotheses of Proposition 5.5 hold with $\nu_{1}=\nu$ and $\nu_{2}=0$. Hence, $\operatorname{Ext}_{G}^{1}\left(L\left(\lambda_{r-1}\right) \otimes L(\nu)^{(1)}, H^{0}(\mu)\right)=0$ for all $\nu \in \Gamma-\{0\}$ and the claim follows. The isomorphism for $G_{r}$ follows by an analogous argument from Theorem 2.7.

5.7. For $r=1$, the difference in the decompositions provided by Theorem 2.1 leads to a slightly different condition for $G\left(\mathbb{F}_{p}\right)$ (compared to Theorem 5.6) while the "same" condition holds for $G_{1}$.

Theorem (A). Let $G$ be a simple simply connected algebraic group with $p \geq 3(h-1)$ and $\lambda, \mu \in X_{1}(T)$. Suppose that for all $\nu \in \Gamma-\{0\}$ :

(i) $\lambda+(p-1) \nu \nless \mu$.

(ii) For any weights $\gamma$ of $L\left(-w_{0} \lambda_{r-1}\right)$ and $\eta$ of $L(\nu), \mu+\gamma+\eta-p \nu$ is not equal to either of the following:

(a) $-\alpha$,

(b) $-p\left\langle\nu+\rho, \alpha^{\vee}\right\rangle \alpha$,

for a simple root $\alpha$. Then $\operatorname{Ext}_{G\left(\mathbb{F}_{p}\right)}^{1}(L(\lambda), L(\mu)) \cong \operatorname{Ext}_{G}^{1}(L(\lambda), L(\mu))$. 
Proof. From Theorem 2.1(c), we have $\operatorname{Ext}_{G\left(\mathbb{F}_{p}\right)}^{1}(L(\lambda), L(\mu)) \cong \operatorname{Ext}_{G}^{1}(L(\lambda), L(\mu)) \oplus R$ where

$$
R=\bigoplus_{\nu \in \Gamma-\{0\}} \operatorname{Ext}_{G}^{1}\left(L(\lambda) \otimes L(\nu)^{(1)}, L(\mu) \otimes L(\nu)\right) .
$$

So it suffices to show that $\operatorname{Ext}_{G}^{1}\left(L(\lambda) \otimes L(\nu)^{(1)}, L(\mu) \otimes L(\nu)\right)=0$ for all $\nu \in \Gamma-\{0\}$. By condition (i), we may apply Lemma 5.5 (with $\nu_{1}=\nu_{2}=\nu$ ) and conclude that there is an embedding

$$
\operatorname{Ext}_{G}^{1}\left(L(\lambda) \otimes L(\nu)^{(1)}, L(\mu) \otimes L(\nu)\right) \hookrightarrow \operatorname{Ext}_{G}^{1}\left(L(\lambda) \otimes L(\nu)^{(1)}, H^{0}(\mu) \otimes L(\nu)\right) .
$$

Then condition (ii) allows us to apply Proposition 5.5 (with $\nu_{1}=\nu_{2}=\nu$ ) and conclude that the latter groups are all zero.

Theorem (B). Let $G$ be a simple simply connected algebraic group with $p \geq$ $3(h-1)$. Let $\lambda, \mu \in X_{1}(T)$. Suppose that for all $\nu \in \Gamma-\{0\}$ :

(i) $\lambda+p \nu \nless \mu$.

(ii) For any weight $\gamma$ of $L\left(-w_{0} \lambda\right), \mu+\gamma-p \nu$ is not equal to either of the following:

(a) $-\alpha$,

(b) $-p\left\langle\nu+\rho, \alpha^{\vee}\right\rangle \alpha$,

for a simple root $\alpha$. Then $\operatorname{Ext}_{G_{1}}^{1}(L(\lambda), L(\mu)) \cong \operatorname{Ext}_{G}^{1}(L(\lambda), L(\mu))$.

Proof. The argument as in Theorem 5.6 for $G_{r}$ with $r \geq 2$ holds for $r=1$ as well.

\section{REFERENCES}

[And1] H.H. Andersen, Extensions of modules for algebraic groups, Amer. J. Math., 106, (1984), 498-504. MR 86g:20056

[And2] H.H. Andersen, Extensions of simple modules for finite Chevalley groups, J. Algebra, 111, (1987), 388-403. MR 89b:20089

[AJ] H.H. Andersen, J.C. Jantzen, Cohomology of induced representations for algebraic groups, Math. Ann., 269, (1984), 487-525. MR 86g:20057

[AJL] H.H. Andersen, J. Jørgensen, P. Landrock, The projective indecomposable modules of SL $\left(2, p^{n}\right)$, Proc. London Math. Soc., (3) 46, (1983), 38-52. MR 84f:20044

[BNP1] C.P. Bendel, D.K. Nakano, C. Pillen, On comparing the cohomology of algebraic groups, finite Chevalley groups, and Frobenius kernels, J. Pure and Appl. Algebra, 163, no. 2, (2001), 119-146. MR 2002e:20094

[BNP2] C.P. Bendel, D.K. Nakano, C. Pillen, Extensions for finite Chevalley groups I., preprint, 2001.

[CPS1] E.T. Cline, B.J. Parshall, L.L. Scott, Cohomology of finite groups of Lie type I, Inst. Hautes Études Sci. Publ. Math., no. 45, (1975), 169-191. MR 53:3134

[CPS2] E.T. Cline, B.J. Parshall, L.L. Scott, Abstract Kazhdan-Lusztig theories, Tohoku Math. J., 45, (1993), 511-534. MR 94k:20079

[CPSK] E. Cline, B. Parshall, L. Scott, W. van der Kallen, Rational and generic cohomology, Invent. Math., 39, (1977), 143-163. MR 55:12737

[F] E.M. Friedlander, Cohomology of irreducible modules with large weights, Proc. Sympos. Pure Math., 47, Part 2, Amer. Math. Soc., Providence, RI, (1987), 187-193. MR 89c:20064

[FP] E.M. Friedlander, B.J. Parshall, Cohomology of infinitesimal and discrete groups. Math. Ann., 273, (1986), no. 3, 353-374. MR 87e:22026

[Hum] J.E. Humphreys, Nonzero Ext ${ }^{1}$ for Chevalley groups (via algebraic groups), J. London Math. Soc., 31, (1985), 463-467. MR 86k:20040

[Jan1] J. C. Jantzen, Representations of Algebraic Groups, Academic Press, Orlando, 1987. MR 89c:20001 
[Jan2] J. C. Jantzen, Über das Dekompositionsverhalten gewisser modularer Darstellungen halbeinfacher Gruppen und ihrer Lie-Algebren, J. Algebra, 49, (1977), 441-469. MR 58:5881

[Jan3] J. C. Jantzen, Darstellungen halbeinfacher Gruppen und ihrer Frobenius-Kerne, J. reine angew. Math, 317, (1980), 157-199. MR 82b:20057

[Jan4] J. C. Jantzen, Zur Reduktion modulo $p$ der Charaktere von Deligne und Lusztig, $J$. Algebra, 70, (1981), 452-474. MR 82m:20045

[Jan5] J. C. Jantzen, First cohomology groups for classical Lie algebras, Progress in Mathematics, 95, Birkhäuser, 1991, 289-315. MR 92e:17024

[Jan6] J. C. Jantzen, Low dimensional representations of reductive groups are semisimple, Algebraic Groups and Lie Groups, ed. G.I. Lehrer, Cambridge University Press, 1997, 255-266. MR 99g:20079

[KLT] S. Kumar, N. Lauritzen, J.F. Thomsen, Frobenius splittings of cotangent bundles of flag varieties, Invent. Math., 136, (1999), 603-621. MR 2000g:20088

[Pil] C. Pillen, Generic patterns for extensions of simple modules for finite Chevalley groups, J. Algebra, 212, (1999), 419-427. MR 2000d:20016

[Ye1] J. Ye, Extensions of simple modules for the group $\operatorname{Sp}(4, K)$, J. London Math. Soc (2), 41, (1990), no. 1, 56-62. MR 91j:20105a

[Ye2] J. Ye, Extensions of simple modules for $G_{2}(p)$, Comm. Alg., 22, (1994), no. 8, 2771-2802. MR 95e:20019

[Yeh] S. Yehia, Extensions of simple modules for the universal Chevalley group and its parabolic subgroups, Ph.D. Thesis, (1982), Warwick University.

Department of Mathematics, Statistics and Computer Science, University of Wisconsin-Stout, Menomonie, Wisconsin 54751

E-mail address: bendelc@uwstout.edu

Department of Mathematics, University of Georgia, Athens, Georgia 30602

E-mail address: nakano@math.uga.edu

Department of Mathematics and Statistics, University of South Alabama, Mobile, Alabama 36688

E-mail address: pillen@jaguar1.usouthal.edu 